\title{
On $\epsilon$-solutions for robust fractional optimization problems
}

\author{
Jae Hyoung Lee and Gue Myung Lee*
}

${ }^{*}$ Correspondence: gmlee@pknu.ac.kr

Department of Applied

Mathematics, Pukyong National

University, 45, Yongso-ro, Nam-Gu,

Busan, 608-737, Korea

\begin{abstract}
We consider $\epsilon$-solutions (approximate solutions) for a fractional optimization problem in the face of data uncertainty. Using robust optimization approach (worst-case approach), we establish optimality theorems and duality theorems for $\epsilon$-solutions for the fractional optimization problem. Moreover, we give an example illustrating our duality theorems.
\end{abstract}

MSC: Primary 90C25; 90C32; secondary 90C46

Keywords: fractional programming under uncertainty; convex programming under uncertainty; strong duality; robust optimization

\section{Introduction}

A robust fractional optimization problem is to optimize an objective fractional function over the constrained set defined by functions with data uncertainty.

To get the $\epsilon$-solution (approximate solution), many authors have established $\epsilon$-optimality conditions and $\epsilon$-duality theorems for several kinds of optimization problems [1-7]. Especially, Lee and Lee [8] gave an $\epsilon$-duality theorems for a convex semidefinite optimization problem with conic constraints. Also, they [9] established optimality theorems and duality theorems for $\epsilon$-solutions for convex optimization problems with uncertainty data.

In [10-15], many authors have treated fractional programming problems in the absence of data uncertainty. Recently, many authors have studied robust optimization problems [9, 16-21]. Very recently, Jeyakumar and Li [22] established duality theorems for a fractional programming problem in the face of data uncertainty via robust optimization.

The purpose of the paper is to extend the $\epsilon$-optimality theorems and $\epsilon$-duality theorems in [9] to fractional optimization problems with uncertainty data.

Consider the following standard form of fractional programming problem with a geometric constraint set:

$$
\begin{array}{ll}
\text { (FP) } \quad \min \frac{f(x)}{g(x)} \\
\text { s.t. } & h_{i}(x) \leqq 0, i=1, \ldots, m, \\
& x \in C,
\end{array}
$$

where $f, h_{i}: \mathbb{R}^{n} \rightarrow \mathbb{R}, i=1, \ldots, m$, are convex functions, $C$ is a closed convex cone of $\mathbb{R}^{n}$, and $g: \mathbb{R}^{n} \rightarrow \mathbb{R}$ is a concave function such that, for any $x \in C, f(x) \geqq 0$ and $g(x)>0$.

@2014 Lee and Lee; licensee Springer. This is an Open Access article distributed under the terms of the Creative Commons Attribution License (http://creativecommons.org/licenses/by/2.0), which permits unrestricted use, distribution, and reproduction in any medium, provided the original work is properly cited. 
The fractional programming problem (FP) in the face of data uncertainty in the constraints can be captured by the problem:

$$
\begin{array}{ll}
(\mathrm{UFP}) \quad \min & \max _{(u, v) \in \mathcal{U} \times \mathcal{V}} \frac{f(x, u)}{g(x, v)} \\
\text { s.t. } & h_{i}\left(x, w_{i}\right) \leqq 0, i=1, \ldots, m, \\
& x \in C,
\end{array}
$$

where $f: \mathbb{R}^{n} \times \mathbb{R}^{p} \rightarrow \mathbb{R}, h_{i}: \mathbb{R}^{n} \times \mathbb{R}^{q} \rightarrow \mathbb{R}, f(\cdot, u)$ and $h_{i}\left(\cdot, w_{i}\right)$ are convex, and $g: \mathbb{R}^{n} \times$ $\mathbb{R}^{p} \rightarrow \mathbb{R}, g(\cdot, v)$ is concave, and $u \in \mathbb{R}^{p}, v \in \mathbb{R}^{p}$, and $w_{i} \in \mathbb{R}^{q}$ are uncertain parameters which belong to the convex and compact uncertainty sets $\mathcal{U} \subset \mathbb{R}^{p}, \mathcal{V} \subset \mathbb{R}^{p}$, and $\mathcal{W}_{i} \subset \mathbb{R}^{q}, i=$ $1, \ldots, m$, respectively.

We study $\epsilon$-optimality theorems and $\epsilon$-duality theorems for the uncertain fractional programming model problem (UFP) by examining its robust (worst-case) counterpart [18]:

$$
\begin{array}{ll}
\text { (RFP) } \quad \min & \max _{(u, v) \in \mathcal{U} \times \mathcal{V}} \frac{f(x, u)}{g(x, v)} \\
\text { s.t. } & h_{i}\left(x, w_{i}\right) \leqq 0, \forall w_{i} \in \mathcal{W}_{i}, i=1, \ldots, m, \\
& x \in C .
\end{array}
$$

Clearly, $A:=\left\{x \in C \mid h_{i}\left(x, w_{i}\right) \leqq 0, \forall w_{i} \in \mathcal{W}_{i}, i=1, \ldots, m\right\}$ is a feasible set of (RFP).

Let $\epsilon \geqq 0$. Then $\bar{x}$ is called an $\epsilon$-solution of (RFP) if, for any $x \in A$,

$$
\max _{(u, v) \in \mathcal{U} \times \mathcal{V}} \frac{f(x, u)}{g(x, v)} \geqq \max _{(u, v) \in \mathcal{U} \times \mathcal{V}} \frac{f(\bar{x}, u)}{g(\bar{x}, v)}-\epsilon .
$$

Using the parametric approach, we transform the problem (RFP) into the robust nonfractional convex optimization problem $(\mathrm{RNCP})_{r}$ with a parametric $r \in \mathbb{R}_{+}$:

$$
\begin{aligned}
& (\mathrm{RNCP})_{r} \quad \min \max _{u \in \mathcal{U}} f(x, u)-r \min _{v \in \mathcal{V}} g(x, v) \\
& \text { s.t. } h_{i}\left(x, w_{i}\right) \leqq 0, \forall w_{i} \in \mathcal{W}_{i}, i=1, \ldots, m, \\
& \quad x \in C .
\end{aligned}
$$

Let $\epsilon \geqq 0$. Then $\bar{x}$ is called an $\epsilon$-solution of (RNCP) $)_{r}$ if, for any $x \in A$,

$$
\max _{u \in \mathcal{U}} f(x, u)-r \min _{v \in \mathcal{V}} g(x, v) \geqq \max _{u \in \mathcal{U}} f(\bar{x}, u)-r \min _{v \in \mathcal{V}} g(\bar{x}, v)-\epsilon .
$$

In this paper, we consider $\epsilon$-solutions for (RFP), and we establish optimality theorems and duality theorems for $\epsilon$-solutions for the robust fractional optimization problem. Moreover, we give an example for our duality theorems.

\section{Preliminaries}

Let us first recall some notation and preliminary results which will be used throughout this paper. $\mathbb{R}^{n}$ denotes the Euclidean space with dimension $n$. The nonnegative orthant of $\mathbb{R}^{n}$ is denoted by $\mathbb{R}_{+}^{n}$ and is defined by $\mathbb{R}_{+}^{n}:=\left\{\left(x_{1}, \ldots, x_{n}\right) \in \mathbb{R}^{n}: x_{i} \geq 0\right\}$. We say the $\operatorname{set} A$ is convex 
whenever $\mu a_{1}+(1-\mu) a_{2} \in A$ for all $\mu \in[0,1], a_{1}, a_{2} \in A$. A function $f: \mathbb{R}^{n} \rightarrow \mathbb{R} \cup\{+\infty\}$ is said to be convex if, for all $\mu \in[0,1]$,

$$
f((1-\mu) x+\mu y) \leq(1-\mu) f(x)+\mu f(y)
$$

for all $x, y \in \mathbb{R}^{n}$. The function $f$ is said to be concave whenever $-f$ is convex. Let $g: \mathbb{R}^{n} \rightarrow$ $\mathbb{R} \cup\{+\infty\}$ be a convex function. The subdifferential of $g$ at $a \in \operatorname{dom} g$ is defined by

$$
\partial g(a):=\left\{v \in \mathbb{R}^{n} \mid g(x) \geqq g(a)+\langle v, x-a\rangle \forall x \in \operatorname{dom} g\right\},
$$

where $\langle\cdot, \cdot\rangle$ is the inner product on $\mathbb{R}^{n}$ and $\operatorname{dom} g:=\left\{x \in \mathbb{R}^{n}: g(x)<+\infty\right\}$. Let $\epsilon \geqq 0$. Then the $\epsilon$-subdifferential of $g$ at $a \in \operatorname{dom} g$ is defined by

$$
\partial_{\epsilon} g(a):=\left\{v \in \mathbb{R}^{n} \mid g(x) \geqq g(a)+\langle v, x-a\rangle-\epsilon \forall x \in \operatorname{dom} g\right\} .
$$

The function $f$ is said to be proper if $f(x)>-\infty$ for all $x \in \mathbb{R}^{n}$. We say $f$ is a lower semicontinuous function if $\liminf _{y \rightarrow x} f(y) \geqq f(x)$ for all $x \in \mathbb{R}^{n}$. As usual, for any proper convex function $g$ on $\mathbb{R}^{n}$, its conjugate function $g^{*}: \mathbb{R}^{n} \rightarrow \mathbb{R} \cup\{+\infty\}$ is defined, for any $x^{*} \in \mathbb{R}^{n}$, by $g^{*}\left(x^{*}\right)=\sup \left\{\left\langle x^{*}, x\right\rangle-g(x) \mid x \in \mathbb{R}^{n}\right\}$. The epigraph of a function $g: \mathbb{R}^{n} \rightarrow \mathbb{R} \cup\{+\infty\}$, epi $g$, is defined by epi $g=\left\{(x, r) \in \mathbb{R}^{n} \times \mathbb{R} \mid g(x) \leqq r\right\}$. We denote the convex hull of a subset $A$ of $\mathbb{R}^{n}$ by $\operatorname{co} A$, and denote the closure of the set $A$ by cl $A$. Let $C$ be a closed convex set in $\mathbb{R}^{n}$ and $x \in C$. Then the normal cone $N_{C}(x)$ to $C$ at $x$ is defined by

$$
N_{C}(x)=\left\{v \in \mathbb{R}^{n} \mid\langle v, y-x\rangle \leqq 0 \text {, for all } y \in C\right\},
$$

and we let $\epsilon \geqq 0$, then the $\epsilon$-normal cone $N_{C}^{\epsilon}(x)$ to $C$ at $x$ is defined by

$$
N_{C}^{\epsilon}(x)=\left\{v \in \mathbb{R}^{n} \mid\langle v, y-x\rangle \leqq \epsilon, \text { for all } y \in C\right\}
$$

When $C$ is a closed convex cone in $\mathbb{R}^{n}$, we denote $N_{C}(0)$ by $C^{*}$ and call it the negative dual cone of $C$.

Proposition 2.1 [23] Let $f: \mathbb{R}^{n} \rightarrow \mathbb{R}$ be a convex function and let $\delta_{C}$ be the indicator function with respect to a closed convex subset $C$ of $\mathbb{R}^{n}$, that is, $\delta_{C}(x)=0$ if $x \in C$, and $\delta_{C}(x)=+\infty$ if $x \notin$ C. Let $\epsilon \geqq 0$. Then

$$
\partial_{\epsilon}\left(f+\delta_{C}\right)(\bar{x})=\bigcup_{\substack{\epsilon_{0} \geqq 0, \epsilon_{1} \geqq 0 \\ \epsilon_{0}+\epsilon_{1}=\epsilon}}\left\{\partial_{\epsilon_{0}} f(\bar{x})+\partial_{\epsilon_{1}} \delta_{C}(\bar{x})\right\} .
$$

Proposition 2.2 $[24,25]$ If $f: \mathbb{R}^{n} \rightarrow \mathbb{R} \cup\{+\infty\}$ is a proper lower semicontinuous convex function and if $a \in \operatorname{dom} f:=\left\{x \in \mathbb{R}^{n} \mid f(x)<+\infty\right\}$, then

$$
\text { epi } f^{*}=\bigcup_{\epsilon \geqq 0}\left\{(v,\langle v, a\rangle+\epsilon-f(a)) \mid v \in \partial_{\epsilon} f(a)\right\} \text {. }
$$

Proposition 2.3 [26] Let $f: \mathbb{R}^{n} \rightarrow \mathbb{R}$ be a convex function and $g: \mathbb{R}^{n} \rightarrow \mathbb{R} \cup\{+\infty\}$ be a proper lower semicontinuous convex function. Then

$$
\operatorname{epi}(f+g)^{*}=\operatorname{epi} f^{*}+\text { epi } g^{*}
$$


Moreover, iff, $g: \mathbb{R}^{n} \rightarrow \mathbb{R} \cup\{+\infty\}$ are proper lower semicontinuous convex functions, and if $\operatorname{dom} f \cap \operatorname{dom} g \neq \emptyset$, then

$$
\mathrm{epi}(f+g)^{*}=\operatorname{cl}\left(\text { epi } f^{*}+\text { epi } g^{*}\right)
$$

Proposition 2.4 [22, 26] Let $h_{i}: \mathbb{R}^{n} \rightarrow \mathbb{R} \cup\{+\infty\}, i \in I$ (where I is an arbitrary index set), be a proper lower semicontinuous convex function. Suppose that there exists $x_{0} \in \mathbb{R}^{n}$ such that $\sup _{i \in I} h_{i}\left(x_{0}\right)<+\infty$. Then

$$
\operatorname{epi}\left(\sup _{i \in I} h_{i}\right)^{*}=\operatorname{cl}\left(\operatorname{co} \bigcup_{i \in I} \operatorname{epi} h_{i}^{*}\right)
$$

Proposition 2.5 [23] Let $h_{i}: \mathbb{R}^{n} \rightarrow \mathbb{R} \cup\{+\infty\}, i=1, \ldots, m$, be proper lower semicontinuous convex functions. Let $\epsilon \geqq 0$. If $\bigcup_{i=1}^{m} r i \operatorname{dom} h_{i} \neq \emptyset$, where $r i \operatorname{dom} h_{i}$ is the relative interior of $\operatorname{dom} h_{i}$, then for all $x \in \bigcup_{i=1}^{n} \operatorname{dom} h_{i}$,

$$
\partial_{\epsilon}\left(\sum_{i=1}^{m} h\right)(x)=\bigcup\left\{\sum_{i=1}^{m} \partial_{\epsilon_{i}} h_{i}(x) \mid \epsilon_{i} \geqq 0, i=1, \ldots, m, \sum_{i=1}^{m} \epsilon_{i}=\epsilon\right\} .
$$

Proposition 2.6 [9] Let $h_{i}: \mathbb{R}^{n} \times \mathbb{R}^{q} \rightarrow \mathbb{R}, i=1, \ldots, m$, be continuous functions such that, for all $w_{i} \in \mathbb{R}^{q}, h_{i}\left(\cdot, w_{i}\right)$ is a convex function and let $C$ be a closed convex cone of $\mathbb{R}^{n}$. Suppose that each $\mathcal{W}_{i}, i=1, \ldots, m$, is compact and convex, and there exists $x_{0} \in C$ such that $h_{i}\left(x_{0}, w_{i}\right)<0$, for all $w_{i} \in \mathcal{W}_{i}, i=1, \ldots, m$. Then

$$
\bigcup_{w_{i} \in \mathcal{W}_{i}, \lambda_{i} \geqq 0} \operatorname{epi}\left(\sum_{i=1}^{m} \lambda_{i} h_{i}\left(\cdot, w_{i}\right)\right)^{*}+C^{*} \times \mathbb{R}_{+}
$$

is closed.

Proposition 2.7 [9] Let $h_{i}: \mathbb{R}^{n} \times \mathbb{R}^{q} \rightarrow \mathbb{R}, i=1, \ldots, m$, be continuous functions and let $C$ be a closed convex cone of $\mathbb{R}^{n}$. Suppose that each $\mathcal{W}_{i} \subseteq \mathbb{R}^{q}, i=1, \ldots, m$, is convex, for all $w_{i} \in \mathbb{R}^{q}, h_{i}\left(\cdot, w_{i}\right)$ is a convex function, and, for each $x \in \mathbb{R}^{n}, h_{i}(x, \cdot)$ is concave on $\mathcal{W}_{i}$. Then

$$
\bigcup_{w_{i} \in \mathcal{W}_{i}, \lambda_{i} \geqq 0} \operatorname{epi}\left(\sum_{i=1}^{m} \lambda_{i} h_{i}\left(\cdot, w_{i}\right)\right)^{*}+C^{*} \times \mathbb{R}_{+}
$$

is convex.

Now we give the following relation between the $\epsilon$-solutions of (RFP) and (RNCP) $)_{\bar{r}}$.

Lemma 2.1 Let $\bar{x} \in A$ and let $\epsilon \geqq 0$. If $\max _{(u, v) \in \mathcal{U} \times \mathcal{V}} \frac{f(\bar{x}, u)}{g(\bar{x}, v)}-\epsilon \geqq 0$, then the following statements are equivalent:

(i) $\bar{x}$ is an $\epsilon$-solution of (RFP);

(ii) $\bar{x}$ is an $\bar{\epsilon}$-solution of $(\mathrm{RNCP})_{\bar{r}}$, where $\bar{r}=\max _{(u, v) \in \mathcal{U} \times \mathcal{V}} \frac{f(\bar{x}, u)}{g(\bar{x}, v)}-\epsilon$ and $\bar{\epsilon}=\epsilon \min _{v \in \mathcal{V}} g(\bar{x}, v)$. 
Proof $(\Rightarrow)$ Let $\bar{x} \in A$ be an $\epsilon$-solution of (RFP). Then for any $x \in A, \max _{(u, v) \in \mathcal{U} \times \mathcal{V}} \frac{f(x, u)}{g(x, v)} \geqq$ $\max _{(u, v) \in \mathcal{U} \times \mathcal{V}} \frac{f(\bar{x}, u)}{g(\bar{x}, v)}-\epsilon$. Put $\bar{r}=\max _{(u, v) \in \mathcal{U} \times \mathcal{V}} \frac{f(\bar{x}, u)}{g(\bar{x}, v)}-\epsilon$ and $\bar{\epsilon}=\epsilon \min _{v \in \mathcal{V}} g(\bar{x}, v)$. Then we have, for any $x \in A, \max _{u \in \mathcal{U}} f(x, u)-\min _{v \in \mathcal{V}} \bar{r} g(x, v) \geqq 0$. Since $\max _{u \in \mathcal{U}} f(\bar{x}, u)-\bar{r} \min _{v \in \mathcal{V}} g(\bar{x}, v)-$ $\epsilon \min _{v \in \mathcal{V}} g(\bar{x}, v)=0$, for any $x \in A$,

$$
\begin{aligned}
\max _{u \in \mathcal{U}} f(x, u)-\bar{r} \min _{v \in \mathcal{V}} g(x, v) & \geqq \max _{u \in \mathcal{U}} f(\bar{x}, u)-\bar{r} \min _{v \in \mathcal{V}} g(\bar{x}, v)-\epsilon \min _{v \in \mathcal{V}} g(\bar{x}, v) \\
& =\max _{u \in \mathcal{U}} f(\bar{x}, u)-\bar{r} \min _{v \in \mathcal{V}} g(\bar{x}, v)-\bar{\epsilon} .
\end{aligned}
$$

Hence $\bar{x}$ is an $\bar{\epsilon}$-solution of $(\mathrm{RNCP})_{\bar{r}}$.

$(\Leftarrow)$ Let $\bar{x} \in A$ be an $\bar{\epsilon}$-solution of $(\mathrm{RNCP})_{\bar{r}}$. Then for any $x \in A, \max _{u \in \mathcal{U}} f(x, u)-$ $\bar{r} \min _{v \in \mathcal{V}} g(x, v) \geqq \max _{u \in \mathcal{U}} f(\bar{x}, u)-\bar{r} \min _{v \in \mathcal{V}} g(\bar{x}, v)-\bar{\epsilon}$. Since $\max _{u \in \mathcal{U}} f(\bar{x}, u)-\bar{r} \min _{v \in \mathcal{V}} g(\bar{x}$, $v)-\epsilon \min _{v \in \mathcal{V}} g(\bar{x}, v)=0$, for any $x \in A, \max _{u \in \mathcal{U}} f(x, u)-\bar{r} \min _{v \in \mathcal{V}} g(x, v) \geqq 0$. So, we have $\max _{(u, v) \in \mathcal{U} \times \mathcal{V}} \frac{f(x, u)}{g(x, v)} \geqq \bar{r}$. Since $\bar{r}=\max _{(u, v) \in \mathcal{U} \times \mathcal{V}} \frac{f(\bar{x}, u)}{g(\bar{x}, v)}-\epsilon$,

$$
\max _{(u, v) \in \mathcal{U} \times \mathcal{V}} \frac{f(x, u)}{g(x, v)} \geqq \max _{(u, v) \in \mathcal{U} \times \mathcal{V}} \frac{f(\bar{x}, u)}{g(\bar{x}, v)}-\epsilon
$$

Hence $\bar{x}$ is an $\epsilon$-solution of (RFP).

\section{$3 \epsilon$-Optimality theorems}

In this section, we establish $\epsilon$-optimality theorems for $\epsilon$-solutions for the robust fractional optimization problem.

Now we give the following lemma which is the robust version of Farkas lemma for nonfractional convex functions.

Lemma 3.1 Let $f: \mathbb{R}^{n} \times \mathbb{R}^{p} \rightarrow \mathbb{R}$ and $h_{i}: \mathbb{R}^{n} \times \mathbb{R}^{q} \rightarrow \mathbb{R}, i=1, \ldots, m$, be functions such that, for any $u \in \mathcal{U}, f(\cdot, u)$ and, for each $w_{i} \in \mathcal{W}_{i}, h_{i}\left(\cdot, w_{i}\right)$ are convex functions, and, for any $x \in \mathbb{R}^{n}, f(x, \cdot)$ is concave function. Let $g: \mathbb{R}^{n} \times \mathbb{R}^{q} \rightarrow \mathbb{R}$ be a function such that, for any $v \in \mathcal{V}, g(\cdot, v)$ is a concave function, and, for all $x \in \mathbb{R}, g(x, \cdot)$ is a convex function. Let $\mathcal{U} \subset \mathbb{R}^{p}$, $\mathcal{V} \subset \mathbb{R}^{p}$, and $\mathcal{W}_{i} \subset \mathbb{R}^{q}, i=1, \ldots, m$ be convex and compact sets. Let $r \geqq 0$ and let $C$ be $a$ closed convex cone of $\mathbb{R}^{n}$. Assume that $A:=\left\{x \in C \mid h_{i}\left(x, w_{i}\right) \leqq 0, \forall w_{i} \in \mathcal{W}_{i}, i=1, \ldots, m\right\} \neq \emptyset$. Then the following statements are equivalent:

(i) $\left\{x \in C \mid h_{i}\left(x, w_{i}\right) \leqq 0, \forall w_{i} \in \mathcal{W}_{i}, i=1, \ldots, m\right\} \subseteq\left\{x \in \mathbb{R}^{n} \mid\right.$

$$
\left.\max _{u \in \mathcal{U}} f(x, u)-r \min _{v \in \mathcal{V}} g(x, v) \geqq 0\right\} \text {; }
$$

(ii) there exist $\bar{u} \in \mathcal{U}$ and $\bar{v} \in \mathcal{V}$ such that

$$
\left\{x \in C \mid h_{i}\left(x, w_{i}\right) \leqq 0, \forall w_{i} \in \mathcal{W}_{i}, i=1, \ldots, m\right\} \subseteq\left\{x \in \mathbb{R}^{n} \mid f(x, \bar{u})-r g(x, \bar{v}) \geqq 0\right\}
$$

(iii)

$$
\begin{aligned}
(0,0) \in & \bigcup_{u \in \mathcal{U}} \operatorname{epi}(f(\cdot, u))^{*}+\bigcup_{v \in \mathcal{V}} \operatorname{epi}(-r g(\cdot, v))^{*} \\
& +\operatorname{clco}\left(\bigcup_{w_{i} \in \mathcal{W}_{i}, \lambda_{i} \geqq 0} \operatorname{epi}\left(\sum_{i=1}^{m} \lambda_{i} h_{i}\left(\cdot, w_{i}\right)\right)^{*}+C^{*} \times \mathbb{R}_{+}\right)
\end{aligned}
$$


(iv)

$$
\begin{aligned}
(0,0) \in & \operatorname{epi}\left(\max _{u \in \mathcal{U}} f(\cdot, u)\right)^{*}+\operatorname{epi}\left(-r \min _{v \in \mathcal{V}} g(\cdot, v)\right)^{*} \\
& +\operatorname{clco}\left(\bigcup_{w_{i} \in \mathcal{W}_{i}, \lambda_{i} \geqq 0} \operatorname{epi}\left(\sum_{i=1}^{m} \lambda_{i} h_{i}\left(\cdot, w_{i}\right)\right)^{*}+C^{*} \times \mathbb{R}_{+}\right) .
\end{aligned}
$$

Proof Let $D:=\left\{x \in \mathbb{R}^{n} \mid h_{i}\left(x, w_{i}\right) \leqq 0, \forall w_{i} \in \mathcal{W}_{i}, i=1, \ldots, m\right\}$. Then $A=C \cap D$. We will prove that epi $\delta_{A}^{*}=\operatorname{clco}\left(\bigcup_{w_{i} \in \mathcal{W}_{i}, \lambda_{i} \geqq 0} \operatorname{epi}\left(\sum_{i=1}^{m} \lambda_{i} h_{i}\left(\cdot, w_{i}\right)\right)^{*}+C^{*} \times \mathbb{R}_{+}\right)$. For any $x \in \mathbb{R}^{n}$,

$$
\delta_{A}(x)=\delta_{C}(x)+\delta_{D}(x) \quad \text { and } \quad \delta_{D}(x)=\sup _{w_{i} \in \mathcal{W}_{i}, \lambda_{i} \geqq 0} \sum_{i=1}^{m} \lambda_{i} h_{i}\left(x, w_{i}\right)
$$

Thus, by Propositions 2.3 and 2.4 , we have

$$
\begin{aligned}
\operatorname{epi} \delta_{A}^{*} & =\operatorname{epi}\left(\delta_{D}+\delta_{C}\right)^{*}=\operatorname{cl}\left(\operatorname{epi} \delta_{D}^{*}+\operatorname{epi} \delta_{C}^{*}\right) \\
& =\operatorname{cl}\left(\operatorname{epi}\left(\sup _{w_{i} \in \mathcal{W}_{i}, \lambda_{i} \geqq 0} \sum_{i=1}^{m} \lambda_{i} h_{i}\left(\cdot, w_{i}\right)\right)^{*}+\operatorname{epi} \delta_{C}^{*}\right) \\
& =\operatorname{cl}\left(\operatorname{clco} \bigcup_{w_{i} \in \mathcal{W}_{i}, \lambda_{i} \geqq 0} \operatorname{epi}\left(\sum_{i=1}^{m} \lambda_{i} h_{i}\left(\cdot, w_{i}\right)\right)^{*}+\operatorname{epi} \delta_{C}^{*}\right) \\
& =\operatorname{clco}\left(\bigcup_{w_{i} \in \mathcal{W}_{i}, \lambda_{i} \geqq 0} \operatorname{epi}\left(\sum_{i=1}^{m} \lambda_{i} h_{i}\left(\cdot, w_{i}\right)\right)^{*}+C^{*} \times \mathbb{R}_{+}\right) .
\end{aligned}
$$

[(i) $\Leftrightarrow$ (iv)] Now we assume that the statement (iv) holds. Then, by Proposition 2.3, the statement (iv) is equivalent to

$$
\begin{aligned}
(0,0) & \in \operatorname{epi}\left(\max _{u \in \mathcal{U}} f(\cdot, u)\right)^{*}+\operatorname{epi}\left(-r \min _{v \in \mathcal{V}} g(\cdot, v)\right)^{*}+\operatorname{epi} \delta_{A}^{*} \\
& =\operatorname{epi}\left(\max _{u \in \mathcal{U}} f(\cdot, u)-r \min _{v \in \mathcal{V}} g(\cdot, v)+\delta_{A}\right)^{*} .
\end{aligned}
$$

Equivalently, by definition of epigraph of $\left(\max _{u \in \mathcal{U}} f(\cdot, u)-r \min _{v \in \mathcal{V}} g(\cdot, v)+\delta_{A}\right)^{*}$,

$$
\left(\max _{u \in \mathcal{U}} f(\cdot, u)-r \min _{v \in \mathcal{V}} g(\cdot, v)+\delta_{A}\right)^{*}(0) \leqq 0
$$

From the definition of a conjugate function, for any $x \in \mathbb{R}^{n}$,

$$
\left(\max _{u \in \mathcal{U}} f(\cdot, u)-r \min _{v \in \mathcal{V}} g(\cdot, v)+\delta_{A}\right)(x) \geqq 0
$$

It is equivalent to the statement that, for any $x \in A$,

$$
\max _{u \in \mathcal{U}} f(x, u)-r \min _{v \in \mathcal{V}} g(x, v) \geqq 0
$$


[(ii) $\Leftrightarrow$ (iii)] Now we assume that the statement (iii) holds. Then the statement (iii) is equivalent to

$$
(0,0) \in \bigcup_{u \in \mathcal{U}} \operatorname{epi}(f(\cdot, u))^{*}+\bigcup_{v \in \mathcal{V}} \operatorname{epi}(-r g(\cdot, v))^{*}+\operatorname{epi} \delta_{A}^{*}
$$

It means that there exist $\bar{u} \in \mathcal{U}$ and $\bar{v} \in \mathcal{V}$ such that

$$
(0,0) \in \operatorname{epi}\left(f(\cdot, \bar{u})-r g(\cdot, \bar{v})+\delta_{A}\right)^{*}
$$

It is equivalent to the statement that there exist $\bar{u} \in \mathcal{U}$ and $\bar{v} \in \mathcal{V}$ such that

$$
\left(f(\cdot, \bar{u})-r g(\cdot, \bar{v})+\delta_{A}\right)^{*}(0) \leqq 0 .
$$

From the definition of a conjugate function, there exist $\bar{u} \in \mathcal{U}$ and $\bar{v} \in \mathcal{V}$ such that, for any $x \in \mathbb{R}^{n}$,

$$
\left(f(\cdot, \bar{u})-r g(\cdot, \bar{v})+\delta_{A}\right)(x) \geqq 0 .
$$

It means that there exist $\bar{u} \in \mathcal{U}$ and $\bar{v} \in \mathcal{V}$ such that, for any $x \in A$,

$$
f(x, \bar{u})-r g(x, \bar{v}) \geqq 0 .
$$

$[(\mathrm{iii}) \Leftrightarrow(\mathrm{iv})]$ To get the desired result, it suffices to show that

$$
\begin{aligned}
& \bigcup_{u \in \mathcal{U}} \operatorname{epi}(f(\cdot, u))^{*}=\operatorname{epi}\left(\max _{u \in \mathcal{U}} f(\cdot, u)\right)^{*}, \\
& \bigcup_{v \in \mathcal{V}} \operatorname{epi}(-r g(\cdot, v))^{*}=\operatorname{epi}\left(-r \min _{v \in \mathcal{V}} g(\cdot, v)\right)^{*} .
\end{aligned}
$$

By Proposition 2.4, epi $\left(\max _{u \in \mathcal{U}} f(\cdot, u)\right)^{*}=\operatorname{clco} \bigcup_{u \in \mathcal{U}} \operatorname{epi}(f(\cdot, u))^{*}$. Let $\left(z_{1}, \alpha_{1}\right),\left(z_{2}, \alpha_{2}\right) \in$ $\bigcup_{u \in \mathcal{U}}$ epi $(f(\cdot, u))^{*}$ and let $\mu \in[0,1]$. Then there exist $u_{1}, u_{2} \in \mathcal{U}$ such that $\left(z_{1}, \alpha_{1}\right) \in$ $\operatorname{epi}\left(f\left(\cdot, u_{1}\right)\right)^{*}$ and $\left(z_{2}, \alpha_{2}\right) \in \operatorname{epi}\left(f\left(\cdot, u_{2}\right)\right)^{*}$, that is, $\left(f\left(\cdot, u_{1}\right)\right)^{*}\left(z_{1}\right) \leqq \alpha_{1}$ and $\left(f\left(\cdot, u_{2}\right)\right)^{*}\left(z_{2}\right) \leqq \alpha_{2}$. Using the definition of a conjugate function, we have, for all $x \in \mathbb{R}^{n}$,

$$
\left\langle z_{1}, x\right\rangle-f\left(x, u_{1}\right) \leqq \alpha_{1} \quad \text { and } \quad\left\langle z_{2}, x\right\rangle-f\left(x, u_{2}\right) \leqq \alpha_{2}
$$

Since, for all $x \in \mathbb{R}^{n}, f(x, \cdot)$ is concave, we have $f\left(x, \mu u_{1}+(1-\mu) u_{2}\right) \geqq \mu f\left(x, u_{1}\right)+(1-$ $\mu) f\left(x, u_{2}\right)$, i.e.,

$$
-f\left(x, \mu u_{1}+(1-\mu) u_{2}\right) \leqq-\mu f\left(x, u_{1}\right)-(1-\mu) f\left(x, u_{2}\right)
$$

So, from (3) and (4), we have, for all $x \in \mathbb{R}^{n}$,

$$
\left\langle\mu z_{1}+(1-\mu) z_{2}, x\right\rangle-f\left(x, \mu u_{1}+(1-\mu) u_{2}\right) \leqq \mu \alpha_{1}+(1-\mu) \alpha_{2}
$$


and so $\left(f\left(\cdot, \mu u_{1}+(1-\mu) u_{2}\right)\right)^{*}\left(\mu z_{1}+(1-\mu) z_{2}\right) \leqq \mu \alpha_{1}+(1-\mu) \alpha_{2}$. Hence, we have

$$
\left(\mu z_{1}+(1-\mu) z_{2}, \mu \alpha_{1}+(1-\mu) \alpha_{2}\right) \in \operatorname{epi}\left(f\left(\cdot, \mu u_{1}+(1-\mu) u_{2}\right)\right)^{*}
$$

So, $\bigcup_{u \in \mathcal{U}} \operatorname{epi}(f(\cdot, u))^{*}$ is convex.

Now we show that $\bigcup_{u \in \mathcal{U}} \operatorname{epi}(f(\cdot, u))^{*}$ is closed. Let

$$
\left(z_{n}, \alpha_{n}\right) \in \bigcup_{u \in \mathcal{U}} \operatorname{epi}(f(\cdot, u))^{*}
$$

with $\left(z_{n}, \alpha_{n}\right) \rightarrow\left(z^{*}, \alpha^{*}\right)$ as $n \rightarrow \infty$. Then there exists $u_{n} \in \mathcal{U}$ such that $\left(f\left(\cdot, u_{n}\right)\right)^{*}\left(z_{n}\right) \leqq \alpha_{n}$. Since $\mathcal{U}$ is compact, we may assume that $u_{n} \rightarrow u^{*} \in \mathcal{U}$ as $n \rightarrow \infty$. So, for each $x \in \mathbb{R}^{n}$,

$$
\left\langle z_{n}, x\right\rangle-f\left(x, u_{n}\right) \leqq \alpha_{n}
$$

Since, for all $x \in \mathbb{R}^{n}, f(x, \cdot)$ is concave, $f(x, \cdot)$ is continuous. Passing to the limit as $n \rightarrow \infty$, we get, for each $x \in \mathbb{R}^{n},\left\langle z^{*}, x\right\rangle-f\left(x, u^{*}\right) \leqq \alpha^{*}$. Hence, we have

$$
\left(z^{*}, \alpha^{*}\right) \in \operatorname{epi}\left(f\left(\cdot, u^{*}\right)\right)^{*}
$$

So, $\bigcup_{u \in \mathcal{U}}$ epi $(f(\cdot, u))^{*}$ is closed. Thus, (1) holds.

Moreover, since, for all $x \in \mathbb{R}^{n}, g(x, \cdot)$ is convex and $r \geqq 0$, for all $x \in \mathbb{R}^{n},-r g(x, \cdot)$ is concave. So, similarly, we can prove that (2) holds.

Remark 3.1 Using convex-concave minimax theorem (Corollary 37.3.2 in [27]), we can prove that the statement (i) in Lemma 3.1 is equivalent to the statement (ii) in Lemma 3.1.

Remark 3.2 From proving in Lemma 3.1 that the statement (i) is equivalent to the statement (iv), we see that we can prove the equivalent relation without the assumptions that, for all $x \in \mathbb{R}^{n}, f(x, \cdot)$, and $g(x, \cdot)$ are concave and convex, respectively.

From Lemmas 2.1 and 3.1, we can get the following theorem.

Theorem 3.1 Let $f: \mathbb{R}^{n} \times \mathbb{R}^{p} \rightarrow \mathbb{R}$ and $h_{i}: \mathbb{R}^{n} \times \mathbb{R}^{q} \rightarrow \mathbb{R}, i=1, \ldots, m$, be functions such that, for any $u \in \mathcal{U}, f(\cdot, u)$, and, for each $w_{i} \in \mathcal{W}_{i}, h_{i}\left(\cdot, w_{i}\right)$ are convex functions, and, for any $x \in \mathbb{R}^{n}, f(x, \cdot)$ is concave function. Let $g: \mathbb{R}^{n} \times \mathbb{R}^{p} \rightarrow \mathbb{R}$ be a function such that, for any $v \in \mathcal{V}, g(\cdot, v)$ is a concave function, and, for all $x \in \mathbb{R}^{n}, g(x, \cdot)$ is a convex function. Let $\mathcal{U} \subset \mathbb{R}^{p}, \mathcal{V} \subset \mathbb{R}^{p}$, and $\mathcal{W}_{i} \subset \mathbb{R}^{q}, i=1, \ldots, m$. Let $\bar{x} \in A$ and let $\bar{r}=\max _{(u, v) \in \mathcal{U} \times \mathcal{V}} \frac{f(\bar{x}, u)}{g(\bar{x}, v)}-\epsilon$. Suppose that $\bigcup_{w_{i} \in \mathcal{W}_{i}, \lambda_{i} \geqq 0} \operatorname{epi}\left(\sum_{i=1}^{m} \lambda_{i} h_{i}\left(\cdot, w_{i}\right)\right)^{*}+C^{*} \times \mathbb{R}_{+}$is closed and convex. Then the following statements are equivalent:

(i) $\bar{x}$ is an $\epsilon$-solution of (RFP);

(ii) There exist $\bar{u} \in \mathcal{U}, \bar{v} \in \mathcal{V}, \bar{w}_{i} \in \mathcal{W}_{i}$, and $\bar{\lambda}_{i} \geqq 0, i=1, \ldots, m$ such that, for any $x \in C$,

$$
f(x, \bar{u})-\bar{r} g(x, \bar{v})+\sum_{i=1}^{m} \bar{\lambda}_{i} h_{i}\left(x, \bar{w}_{i}\right) \geqq 0 .
$$


Proof $(\Rightarrow)$ Let $\bar{x}$ be an $\epsilon$-solution of (RFP). Then, by Lemma 2.1, equivalently, $\bar{x}$ is an $\bar{\epsilon}$-solution of $(\mathrm{RNCP})_{\bar{r}}$, where $\bar{r}=\max _{(u, v) \in \mathcal{U} \times \mathcal{V}} \frac{f(\bar{x}, u)}{g(\bar{x}, v)}-\epsilon$ and $\bar{\epsilon}=\epsilon \min _{v \in \mathcal{V}} g(\bar{x}, v)$, that is, for any $x \in A, \max _{u \in \mathcal{U}} f(x, u)-\bar{r} \min _{v \in \mathcal{V}} g(x, v) \geqq \max _{u \in \mathcal{U}} f(\bar{x}, u)-\bar{r} \min _{v \in \mathcal{V}} g(\bar{x}, v)-$ $\epsilon \min _{v \in \mathcal{V}} g(\bar{x}, v)$. Since $\max _{u \in \mathcal{U}} f(\bar{x}, u)-\bar{r} \min _{v \in \mathcal{V}} g(\bar{x}, v)-\epsilon \min _{v \in \mathcal{V}} g(\bar{x}, v)=0$, we have $A \subseteq$ $\left\{x \in C \mid \max _{u \in \mathcal{U}} f(x, u)-\bar{r} \min _{v \in \mathcal{V}} g(x, v) \geqq 0\right\}$. Then, by Lemma 3.1, we have

$$
\begin{aligned}
(0,0) \in \bigcup_{u \in \mathcal{U}} \operatorname{epi}(f(\cdot, u))^{*}+\bigcup_{\nu \in \mathcal{V}} \operatorname{epi}(-\bar{r} g(\cdot, v))^{*} \\
\quad+\operatorname{clco}\left(\bigcup_{w_{i} \in \mathcal{W}_{i}, \lambda_{i} \geqq 0} \operatorname{epi}\left(\sum_{i=1}^{m} \lambda_{i} h_{i}\left(\cdot, w_{i}\right)\right)^{*}+C^{*} \times \mathbb{R}_{+}\right) .
\end{aligned}
$$

Moreover, by assumption,

$$
(0,0) \in \bigcup_{u \in \mathcal{U}} \operatorname{epi}(f(\cdot, u))^{*}+\bigcup_{v \in \mathcal{V}} \operatorname{epi}(-\bar{r} g(\cdot, v))^{*}+\bigcup_{w_{i} \in \mathcal{W}_{i}, \lambda_{i} \geq 0} \operatorname{epi}\left(\sum_{i=1}^{m} \lambda_{i} h_{i}\left(\cdot, w_{i}\right)\right)^{*}+C^{*} \times \mathbb{R}_{+} .
$$

So, there exist $\bar{u} \in \mathcal{U}, \bar{v} \in \mathcal{V}, \bar{w}_{i} \in \mathcal{W}_{i}$, and $\bar{\lambda}_{i} \geqq 0, i=1, \ldots, m$ such that

$$
(0,0) \in \operatorname{epi}(f(\cdot, \bar{u}))^{*}+\operatorname{epi}(-\bar{r} g(\cdot, \bar{v}))^{*}+\operatorname{epi}\left(\sum_{i=1}^{m} \bar{\lambda}_{i} h_{i}\left(\cdot, \bar{w}_{i}\right)\right)^{*}+C^{*} \times \mathbb{R}_{+} .
$$

Then there exist $s \in \mathbb{R}^{n}, \eta \geqq 0, t \in \mathbb{R}^{n}, \mu \geqq 0, z_{i} \in \mathbb{R}^{n}, \rho_{i} \geqq 0, i=1, \ldots, m, c^{*} \in C^{*}$, and $\gamma \in \mathbb{R}_{+}$such that

$$
\begin{aligned}
(0,0)= & \left(s,(f(\cdot, \bar{u}))^{*}(s)+\eta\right)+\left(t,(-\bar{r} g(\cdot, \bar{v}))^{*}(t)+\mu\right) \\
& +\sum_{i=1}^{m}\left(z_{i},\left(\bar{\lambda}_{i} h_{i}\left(\cdot, \bar{w}_{i}\right)\right)^{*}\left(z_{i}\right)+\rho_{i}\right)+\left(c^{*}, \gamma\right) .
\end{aligned}
$$

So, $0=s+t+\sum_{i=1}^{m} z_{i}+c^{*}$ and $0=(f(\cdot, \bar{u}))^{*}(s)+\eta+(-\bar{r} g(\cdot, \bar{v}))^{*}(t)+\mu+\sum_{i=1}^{m}\left(\left(\bar{\lambda}_{i} h_{i}\left(\cdot, \bar{w}_{i}\right)\right)^{*}\left(z_{i}\right)+\right.$ $\left.\rho_{i}\right)+\gamma$. Hence, for any $x \in \mathbb{R}^{n}$,

$$
\begin{aligned}
& -\left\langle\sum_{i=1}^{m} z_{i}, x\right\rangle-\left\langle c^{*}, x\right\rangle-f(x, \bar{u})-(-\bar{r} g(x, \bar{v})) \\
& =\langle s, x\rangle+\langle t, x\rangle-f(x, \bar{u})-(-\bar{r} g(x, \bar{v})) \\
& \leqq \\
& \quad=(f(\cdot, \bar{u}))^{*}(s)+(-\bar{r} g(\cdot, v))^{*}(t) \\
& =-\eta-\mu-\sum_{i=1}^{m}\left(\left(\bar{\lambda}_{i} h_{i}\left(\cdot, \bar{w}_{i}\right)\right)^{*}\left(z_{i}\right)+\rho_{i}\right)-\gamma .
\end{aligned}
$$

Since $\eta \geqq 0, \mu \geqq 0, \rho_{i} \geqq 0, i=1, \ldots, m$, and $c^{*} \in C^{*}$, from (5), for any $x \in C$,

$$
\begin{aligned}
0 \leqq & \left\langle\sum_{i=1}^{m} z_{i}, x\right\rangle+\left\langle c^{*}, x\right\rangle+f(x, \bar{u})+(-\bar{r} g(x, \bar{v}))-\eta-\mu \\
& -\sum_{i=1}^{m}\left(\bar{\lambda}_{i} h_{i}\left(\cdot, \bar{w}_{1}\right)\right)^{*}\left(z_{i}\right)-\sum_{i=1}^{m} \bar{\lambda}_{i} \rho_{i}-\gamma
\end{aligned}
$$




$$
\begin{aligned}
& \leqq\left\langle\sum_{i=1}^{m} z_{i}, x\right\rangle+f(x, \bar{u})-\bar{r} g(x, \bar{v})-\sum_{i=1}^{m}\left(\bar{\lambda}_{i} h_{i}\left(\cdot, \bar{w}_{i}\right)\right)^{*}\left(z_{i}\right) \\
& \leqq f(x, \bar{u})-\bar{r} g(x, \bar{v})+\sum_{i=1}^{m}\left(\bar{\lambda}_{i} h_{i}\left(x, \bar{w}_{i}\right)\right) .
\end{aligned}
$$

$(\Leftarrow)$ Suppose that there exist $\bar{u} \in \mathcal{U}, \bar{v} \in \mathcal{V}, \bar{w}_{i} \in \mathcal{W}_{i}$, and $\bar{\lambda}_{i} \geqq 0, i=1, \ldots, m$, such that, for any $x \in C$,

$$
f(x, \bar{u})-\bar{r} g(x, \bar{v})+\sum_{i=1}^{m} \bar{\lambda}_{i} h_{i}\left(x, \bar{w}_{i}\right) \geqq 0 .
$$

Since $\bar{r}=\max _{(u, v) \in \mathcal{U} \times \mathcal{V}} \frac{f(\bar{x}, u)}{g(\bar{x}, v)}-\epsilon$, we have $\max _{u \in \mathcal{U}} f(\bar{x}, u)-\bar{r} \min _{v \in \mathcal{V}} g(\bar{x}, v)-\epsilon \min _{v \in \mathcal{V}} g(\bar{x}, v)=$ 0 . So, from (6), we have, for any $x \in A$,

$$
\begin{aligned}
\max _{u \in \mathcal{U}} f(x, u)-\bar{r} \min _{v \in \mathcal{V}} g(x, v) & \geqq \max _{u \in \mathcal{U}} f(x, u)-\bar{r} \min _{v \in \mathcal{V}} g(x, v)+\sum_{i=1}^{m} \bar{\lambda}_{i} h_{i}\left(x, \bar{w}_{i}\right) \\
& \geqq f(x, \bar{u})-\bar{r} g(x, \bar{v})+\sum_{i=1}^{m} \bar{\lambda}_{i} h_{i}\left(x, \bar{w}_{i}\right) \\
& \geqq 0 \\
& =\max _{u \in \mathcal{U}} f(\bar{x}, u)-\bar{r} \min _{v \in \mathcal{V}} g(\bar{x}, v)-\epsilon \min _{v \in \mathcal{V}} g(\bar{x}, v) .
\end{aligned}
$$

Hence, for any $x \in A, \max _{u \in \mathcal{U}} f(x, u)-\bar{r} \min _{v \in \mathcal{V}} g(x, v) \geqq \max _{u \in \mathcal{U}} f(\bar{x}, u)-\bar{r} \min _{v \in \mathcal{V}} g(\bar{x}, v)-$ $\epsilon \min _{v \in \mathcal{V}} g(\bar{x}, v)$. It means that $\bar{x}$ is an $\bar{\epsilon}$-solution of (RNCP) $)_{\bar{r}}$. Thus, by Lemma $2.1, \bar{x}$ is an $\epsilon$-solution of (RFP).

Using Remark 3.2 and Lemmas 2.1 and 3.1, we can obtain the following characterization of an $\epsilon$-solution for (RFP).

Theorem 3.2 ( $\epsilon$-Optimality theorem) Let $f: \mathbb{R}^{n} \times \mathbb{R}^{p} \rightarrow \mathbb{R}$ and $h_{i}: \mathbb{R}^{n} \times \mathbb{R}^{q} \rightarrow \mathbb{R}, i=$ $1, \ldots, m$, be functions such that, for any $u \in \mathcal{U}, f(\cdot, u)$, and, for each $w_{i} \in \mathcal{W}_{i}, h_{i}\left(\cdot, w_{i}\right)$ are convex functions. Let $g: \mathbb{R}^{n} \times \mathbb{R}^{p} \rightarrow \mathbb{R}$ be a function such that, for any $v \in \mathcal{V}, g(\cdot, v)$ is a concave function. Let $\mathcal{U} \subset \mathbb{R}^{p}, \mathcal{V} \subset \mathbb{R}^{p}$, and $\mathcal{W}_{i} \subset \mathbb{R}^{q}, i=1, \ldots$, m. Let $\bar{x} \in A$ and let $\epsilon \geqq 0$. Let $\bar{r}=\max _{(u, v) \in \mathcal{U} \times \mathcal{V}} \frac{f(\bar{x}, u)}{g(\bar{x}, v)}-\epsilon$. If $\max _{(u, v) \in \mathcal{U} \times \mathcal{V}} \frac{f(\bar{x}, u)}{g(\bar{x}, v)}<\epsilon$, then $\bar{x}$ is an $\epsilon$-solution of (RFP). If $\max _{(u, v) \in \mathcal{U} \times \mathcal{V}} \frac{f(\bar{x}, u)}{g(\bar{x}, v)} \geqq \epsilon$ and

$$
\bigcup_{w_{i} \in \mathcal{W}_{i}, \lambda_{i} \geqq 0} \operatorname{epi}\left(\sum_{i=1}^{m} \lambda_{i} h_{i}\left(\cdot, w_{i}\right)\right)^{*}+C^{*} \times \mathbb{R}_{+}
$$

is closed and convex, then the following statements are equivalent:

(i) $\bar{x}$ is an $\epsilon$-solution of (RFP); 
(ii) there exist $\bar{w}_{i} \in \mathcal{W}_{i}$ and $\bar{\lambda}_{i} \geqq 0, i=1, \ldots, m, \epsilon_{0}^{1} \geqq 0, \epsilon_{0}^{2} \geqq 0$, and $\epsilon_{i} \geqq 0, i=1, \ldots, m+1$ such that

$$
\begin{aligned}
& 0 \in \partial_{\epsilon_{0}^{1}}\left(\max _{u \in \mathcal{U}} f(\cdot, u)\right)(\bar{x})+\partial_{\epsilon_{0}^{2}}\left(-\bar{r} \min _{v \in \mathcal{V}} g(\cdot, v)\right)(\bar{x}) \\
& +\sum_{i=1}^{m} \partial_{\epsilon_{i}}\left(\bar{\lambda}_{i} h_{i}\left(\cdot, \bar{w}_{i}\right)\right)(\bar{x})+N_{C}^{\epsilon_{m+1}}(\bar{x}), \\
& \max _{u \in \mathcal{U}} f(\bar{x}, u)-\bar{r} \min _{v \in \mathcal{V}} g(\bar{x}, v)=\epsilon \min _{v \in \mathcal{V}} g(\bar{x}, v) \quad \text { and } \\
& \epsilon_{0}^{1}+\epsilon_{0}^{2}+\sum_{i=1}^{m+1} \epsilon_{i}-\epsilon \min _{\nu \in \mathcal{V}} g(\bar{x}, v)=\sum_{i=1}^{m} \bar{\lambda}_{i} h_{i}\left(\bar{x}, \bar{w}_{i}\right) .
\end{aligned}
$$

Proof $[(\mathrm{i}) \Rightarrow(\mathrm{ii})]$ We assume that $\bar{x}$ is an $\epsilon$-solution of (RFP). Then, by Lemma $2.1, \bar{x}$ is an $\bar{\epsilon}$-solution of $(\mathrm{RNCP})_{\bar{r}}$, where $\bar{r}=\max _{(u, v) \in \mathcal{U} \times \mathcal{V}} \frac{f(\bar{x}, u)}{g(\bar{x}, v)}-\epsilon$ and $\bar{\epsilon}=\epsilon \min _{v \in \mathcal{V}} g(\bar{x}, v)$, that is, for any $x \in A, \max _{u \in \mathcal{U}} f(x, u)-\bar{r} \min _{v \in \mathcal{V}} g(x, v) \geqq \max _{u \in \mathcal{U}} f(\bar{x}, u)-\bar{r} \min _{v \in \mathcal{V}} g(\bar{x}, v)-$ $\epsilon \min _{v \in \mathcal{V}} g(\bar{x}, v)$. Since $\max _{u \in \mathcal{U}} f(\bar{x}, u)-\bar{r} \min _{v \in \mathcal{V}} g(\bar{x}, v)-\epsilon \min _{v \in \mathcal{V}} g(\bar{x}, v)=0$, we have $A \subseteq$ $\left\{x \in C \mid \max _{u \in \mathcal{U}} f(x, u)-\bar{r} \min _{v \in \mathcal{V}} g(x, v) \geqq 0\right\}$. By Lemma 3.1,

$$
\begin{aligned}
(0,0) \in & \operatorname{epi}\left(\max _{u \in \mathcal{U}} f(\cdot, u)\right)^{*}+\operatorname{epi}\left(-\bar{r} \min _{v \in \mathcal{V}} g(\cdot, v)\right)^{*} \\
& +\operatorname{clco}\left(\bigcup_{w_{i} \in \mathcal{W}_{i}, \lambda_{i} \geqq 0} \operatorname{epi}\left(\sum_{i=1}^{m} \lambda_{i} h_{i}\left(\cdot, w_{i}\right)\right)^{*}+C^{*} \times \mathbb{R}_{+}\right) .
\end{aligned}
$$

By assumption,

$$
\begin{aligned}
(0,0) \in & \operatorname{epi}\left(\max _{u \in \mathcal{U}} f(\cdot, u)\right)^{*}+\operatorname{epi}\left(-\bar{r} \min _{v \in \mathcal{V}} g(\cdot, v)\right)^{*} \\
& +\bigcup_{w_{i} \in \mathcal{W}_{i}, \lambda_{i} \geqq 0} \operatorname{epi}\left(\sum_{i=1}^{m} \lambda_{i} h_{i}\left(\cdot, w_{i}\right)\right)^{*}+C^{*} \times \mathbb{R}_{+} .
\end{aligned}
$$

So, there exist $\bar{w}_{i} \in \mathcal{W}_{i}$ and $\bar{\lambda}_{i} \geqq 0, i=1, \ldots, m$, such that

$$
(0,0) \in \operatorname{epi}\left(\max _{u \in \mathcal{U}} f(\cdot, u)\right)^{*}+\operatorname{epi}\left(-\bar{r} \min _{v \in \mathcal{V}} g(\cdot, v)\right)^{*}+\operatorname{epi}\left(\sum_{i=1}^{m} \bar{\lambda}_{i} h_{i}\left(\cdot, \bar{w}_{i}\right)\right)^{*}+\operatorname{epi} \delta_{C}^{*} .
$$

By Proposition 2.2, we obtain

$$
\begin{aligned}
(0,0) \in & \bigcup_{\epsilon_{0}^{1} \geqq 0}\left\{\left(\xi_{0}^{1},\left\langle\xi_{0}^{1}, \bar{x}\right\rangle+\epsilon_{0}^{1}-\max _{u \in \mathcal{U}} f(\bar{x}, u)\right) \mid \xi_{0}^{1} \in \partial_{\epsilon_{0}^{1}}\left(\max _{u \in \mathcal{U}} f(\cdot, u)\right)(\bar{x})\right\} \\
& +\bigcup_{\epsilon_{0}^{2} \geqq 0}\left\{\left(\xi_{0}^{2},\left\langle\xi_{0}^{2}, \bar{x}\right\rangle+\epsilon_{0}^{2}+\bar{r} \min _{v \in \mathcal{V}} g(\bar{x}, v)\right) \mid \xi_{0}^{2} \in \partial_{\epsilon_{0}^{2}}\left(-\bar{r} \min _{v \in \mathcal{V}} g(\cdot, v)\right)(\bar{x})\right\} \\
& +\bigcup_{\epsilon^{*} \geqq 0}\left\{\left(\xi^{*},\left\langle\xi^{*}, \bar{x}\right\rangle+\epsilon^{*}-\sum_{i=1}^{m} \bar{\lambda}_{i} h_{i}\left(\bar{x}, \bar{w}_{i}\right)\right) \mid \xi^{*} \in \partial_{\epsilon^{*}}\left(\sum_{i=1}^{m} \bar{\lambda}_{i} h_{i}\left(\cdot, \bar{w}_{i}\right)\right)(\bar{x})\right\} \\
& +\bigcup_{\epsilon_{m+1} \geqq 0}\left\{\left(\xi_{m+1},\left\langle\xi_{m+1}, \bar{x}\right\rangle+\epsilon_{m+1}-\delta_{C}(\bar{x})\right) \mid \xi_{m+1} \in \partial_{\epsilon_{m+1}} \delta_{C}(\bar{x})\right\} .
\end{aligned}
$$


So, there exist $\bar{\xi}_{0}^{1} \in \partial_{\epsilon_{0}^{1}}\left(\max _{u \in \mathcal{U}} f(\cdot, u)\right)(\bar{x}), \bar{\xi}_{0}^{2} \in \partial_{\epsilon_{0}^{2}}\left(-\bar{r} \min _{v \in \mathcal{V}} g(\cdot, v)\right)(\bar{x}), \bar{\xi}^{*} \in \partial_{\epsilon^{*}}\left(\sum_{i=1}^{m} \bar{\lambda}_{i} h_{i}(\cdot\right.$, $\left.\left.\bar{w}_{i}\right)\right)(\bar{x}), \bar{\xi}_{m+1} \in \partial_{\epsilon_{m+1}} \delta_{C}(\bar{x}), \epsilon_{0}^{1} \geqq 0, \epsilon_{0}^{2} \geqq 0, \epsilon^{*} \geqq 0$, and $\epsilon_{m+1} \geqq 0$ such that

$$
\begin{aligned}
& 0=\bar{\xi}_{0}^{1}+\bar{\xi}_{0}^{2}+\bar{\xi}^{*}+\bar{\xi}_{m+1} \quad \text { and } \\
& \epsilon_{0}^{1}+\epsilon_{0}^{2}+\epsilon^{*}+\epsilon_{m+1}=\max _{u \in \mathcal{U}} f(\bar{x}, u)-\bar{r} \min _{v \in \mathcal{V}} g(\bar{x}, v)+\sum_{i=1}^{m} \bar{\lambda}_{i} h_{i}\left(\bar{x}, \bar{w}_{i}\right) .
\end{aligned}
$$

By Proposition 2.5, there exist $\bar{\xi}_{0}^{1} \in \partial_{\epsilon_{0}^{1}}\left(\max _{u \in \mathcal{U}} f(\cdot, u)\right)(\bar{x}), \bar{\xi}_{0}^{2} \in \partial_{\epsilon_{0}^{2}}\left(-\bar{r} \min _{v \in \mathcal{V}} g(\cdot, v)\right)(\bar{x}), \bar{\xi}_{i} \in$ $\partial_{\epsilon_{i}}\left(\bar{\lambda}_{i} h_{i}\left(\cdot, \bar{w}_{i}\right)\right)(\bar{x}), \bar{\xi}_{m+1} \in \partial_{\epsilon_{m+1}} \delta_{C}(\bar{x}), \epsilon_{0}^{1} \geqq 0, \epsilon_{0}^{2} \geqq 0, \epsilon_{i} \geqq 0, i=1, \ldots, m$, and $\epsilon_{m+1} \geqq 0$ such that

$$
\begin{aligned}
& 0 \in \partial_{\epsilon_{0}^{1}}\left(\max _{u \in \mathcal{U}} f(\cdot, u)\right)(\bar{x})+\partial_{\epsilon_{0}^{2}}\left(-\bar{r} \min _{v \in \mathcal{V}} g(\cdot, v)\right)(\bar{x}) \\
&+\sum_{i=1}^{m} \partial_{\epsilon_{i}}\left(\bar{\lambda}_{i} h_{i}\left(\cdot, \bar{w}_{i}\right)\right)(\bar{x})+N_{C}^{\epsilon_{m+1}}(\bar{x}) \quad \text { and } \\
& \epsilon_{0}^{1}+\epsilon_{0}^{2}+\sum_{i=1}^{m+1} \epsilon_{i}=\max _{u \in \mathcal{U}} f(\bar{x}, u)-\bar{r} \min _{v \in \mathcal{V}} g(\bar{x}, v)+\sum_{i=1}^{m} \bar{\lambda}_{i} h_{i}\left(\bar{x}, \bar{w}_{i}\right) .
\end{aligned}
$$

Since $\bar{r}=\max _{(u, v) \in \mathcal{U} \times \mathcal{V}} \frac{f(\bar{x}, u)}{g(\bar{x}, v)}-\epsilon$,

$$
\max _{u \in \mathcal{U}} f(\bar{x}, u)-\bar{r} \min _{v \in \mathcal{V}} g(\bar{x}, v)-\epsilon \min _{v \in \mathcal{V}} g(\bar{x}, v)=0
$$

So, (8) holds, and so, from (10) and (11), we have

$$
\begin{gathered}
0 \in \partial_{\epsilon_{0}^{1}}\left(\max _{u \in \mathcal{U}} f(\cdot, u)\right)(\bar{x})+\partial_{\epsilon_{0}^{2}}\left(-\bar{r} \min _{v \in \mathcal{V}} g(\cdot, v)\right)(\bar{x}) \\
+\sum_{i=1}^{m} \partial_{\epsilon_{i}}\left(\bar{\lambda}_{i} h_{i}\left(\cdot, \bar{w}_{i}\right)\right)(\bar{x})+N_{C}^{\epsilon_{m+1}}(\bar{x}) \quad \text { and } \\
\epsilon_{0}^{1}+\epsilon_{0}^{2}+\sum_{i=1}^{m+1} \epsilon_{i}-\epsilon \min _{v \in \mathcal{V}} g(\bar{x}, v)=\sum_{i=1}^{m} \bar{\lambda}_{i} h_{i}\left(\bar{x}, \bar{w}_{i}\right) .
\end{gathered}
$$

Thus the conditions (7) and (9) hold.

[(ii) $\Rightarrow$ (i)] Taking account of the converse of the process for proving (i) $\Rightarrow$ (ii), we can easily check that the statement (ii) $\Rightarrow$ (i) holds.

If for all $x \in \mathbb{R}^{n}, f(x, \cdot)$ is concave, and, for all $x \in \mathbb{R}, g(x, \cdot)$ is convex, then using Lemmas 2.1 and 3.1, we can obtain the following characterization of an $\epsilon$-solution for (RFP).

Theorem 3.3 ( $\epsilon$-Optimality theorem) Let $f: \mathbb{R}^{n} \times \mathbb{R}^{p} \rightarrow \mathbb{R}$ and $h_{i}: \mathbb{R}^{n} \times \mathbb{R}^{q} \rightarrow \mathbb{R}, i=$ $1, \ldots, m$, be functions such that, for any $u \in \mathcal{U}, f(\cdot, u)$, and, for each $w_{i} \in \mathcal{W}_{i}, h_{i}\left(\cdot, w_{i}\right)$ are convex functions, and, for all $x \in \mathbb{R}^{n}, f(x, \cdot)$ is concave function. Let $g: \mathbb{R}^{n} \times \mathbb{R}^{p} \rightarrow \mathbb{R}$ be a function such that, for any $v \in \mathcal{V}, g(\cdot, v)$ is a concave function, and, for all $x \in \mathbb{R}, g(x, \cdot)$ is a convex function. Let $\mathcal{U} \subset \mathbb{R}^{p}, \mathcal{V} \subset \mathbb{R}^{p}$, and $\mathcal{W}_{i} \subset \mathbb{R}^{q}, i=1, \ldots, m$. Let $\bar{x} \in A$ and let $\epsilon \geqq 0$. Let $\bar{r}=\max _{(u, v) \in \mathcal{U} \times \mathcal{V}} \frac{f(\bar{x}, u)}{g(\bar{x}, v)}-\epsilon$. If $\max _{(u, v) \in \mathcal{U} \times \mathcal{V}} \frac{f(\bar{x}, u)}{g(\bar{x}, v)}<\epsilon$, then $\bar{x}$ is an $\epsilon$-solution of (RFP). If 
$\max _{(u, v) \in \mathcal{U} \times \mathcal{V}} \frac{f(\bar{x}, u)}{g(\bar{x}, v)} \geqq \epsilon$ and

$$
\bigcup_{w_{i} \in \mathcal{W}_{i}, \lambda_{i} \geqq 0} \operatorname{epi}\left(\sum_{i=1}^{m} \lambda_{i} h_{i}\left(\cdot, w_{i}\right)\right)^{*}+C^{*} \times \mathbb{R}_{+}
$$

is closed and convex, then the following statements are equivalent:

(i) $\bar{x}$ is an $\epsilon$-solution of (RFP);

(ii) there exist $\bar{u} \in \mathcal{U}, \bar{v} \in \mathcal{V}, \bar{w}_{i} \in \mathcal{W}_{i}, \bar{\lambda}_{i} \geqq 0, i=1, \ldots, m, \epsilon_{0}^{1} \geqq 0, \epsilon_{0}^{2} \geqq 0$, and $\epsilon_{i} \geqq 0$, $i=1, \ldots, m+1$ such that

$$
\begin{aligned}
& 0 \in \partial_{\epsilon_{0}^{1}}(f(\cdot, \bar{u}))(\bar{x})+\partial_{\epsilon_{0}^{2}}(-\bar{r} g(\cdot, \bar{v}))(\bar{x})+\sum_{i=1}^{m} \partial_{\epsilon_{i}}\left(\bar{\lambda}_{i} h_{i}\left(\cdot, \bar{w}_{i}\right)\right)(\bar{x})+N_{C}^{\epsilon_{m+1}}(\bar{x}), \\
& \max _{u \in \mathcal{U}} f(\bar{x}, u)-\min _{v \in \mathcal{V}} \bar{r} g(\bar{x}, v)=\epsilon \min _{v \in \mathcal{V}} g(\bar{x}, v) \text { and } \\
& \epsilon_{0}^{1}+\epsilon_{0}^{2}+\sum_{i=1}^{m+1} \epsilon_{i}-\epsilon \min _{v \in \mathcal{V}} g(\bar{x}, v) \leqq \sum_{i=1}^{m} \bar{\lambda}_{i} h_{i}\left(\bar{x}, \bar{w}_{i}\right)
\end{aligned}
$$

Proof $[(\mathrm{i}) \Rightarrow(\mathrm{ii})]$ Let $\bar{x}$ be an $\epsilon$-solution of (RFP). Then, by Lemma $2.1, \bar{x}$ is an $\bar{\epsilon}$-solution of (RNCP) $)_{\bar{r}}$, where $\bar{r}=\max _{(u, v) \in \mathcal{U} \times \mathcal{V}} \frac{f(\bar{x}, u)}{g(\bar{x}, v)}-\epsilon$ and $\bar{\epsilon}=\epsilon \min _{v \in \mathcal{V}} g(\bar{x}, v)$, that is, for any $x \in A$, $\max _{u \in \mathcal{U}} f(x, u)-\bar{r} \min _{v \in \mathcal{V}} g(x, v) \geqq \max _{u \in \mathcal{U}} f(\bar{x}, u)-\bar{r} \min _{v \in \mathcal{V}} g(\bar{x}, v)-\epsilon \min _{v \in \mathcal{V}} g(\bar{x}, v)$. Since $\max _{u \in \mathcal{U}} f(\bar{x}, u)-\bar{r} \min _{v \in \mathcal{V}} g(\bar{x}, v)-\epsilon \min _{v \in \mathcal{V}} g(\bar{x}, v)=0$, we have $A \subseteq\left\{x \in C \mid \max _{u \in \mathcal{U}} f(x, u)-\right.$ $\left.\bar{r} \min _{v \in \mathcal{V}} g(x, v) \geqq 0\right\}$. By Lemma 3.1,

$$
\begin{aligned}
(0,0) \in & \bigcup_{u \in \mathcal{U}} \operatorname{epi}(f(\cdot, u))^{*}+\bigcup_{v \in \mathcal{V}} \operatorname{epi}(-\bar{r} g(\cdot, v))^{*} \\
& +\operatorname{clco}\left(\bigcup_{w_{i} \in \mathcal{W}_{i}, \lambda_{i} \geqq 0} \operatorname{epi}\left(\sum_{i=1}^{m} \lambda_{i} h_{i}\left(\cdot, w_{i}\right)\right)^{*}+C^{*} \times \mathbb{R}_{+}\right) .
\end{aligned}
$$

By assumption,

$$
(0,0) \in \bigcup_{u \in \mathcal{U}} \operatorname{epi}(f(\cdot, u))^{*}+\bigcup_{\nu \in \mathcal{V}} \operatorname{epi}(-\bar{r} g(\cdot, v))^{*}+\bigcup_{w_{i} \in \mathcal{W}_{i}, \lambda_{i} \geqq 0} \operatorname{epi}\left(\sum_{i=1}^{m} \lambda_{i} h_{i}\left(\cdot, w_{i}\right)\right)^{*}+C^{*} \times \mathbb{R}_{+} .
$$

Since $C^{*} \times \mathbb{R}_{+}=$epi $\delta_{C}^{*}$, there exist $\bar{u} \in \mathcal{U}, \bar{v} \in \mathcal{V}, \bar{w}_{i} \in \mathcal{W}_{i}$, and $\bar{\lambda}_{i} \geqq 0, i=1, \ldots, m$, such that

$$
(0,0) \in \operatorname{epi}(f(\cdot, \bar{u}))^{*}+\operatorname{epi}(-\bar{r} g(\cdot, \bar{v}))^{*}+\operatorname{epi}\left(\sum_{i=1}^{m} \bar{\lambda}_{i} h_{i}\left(\cdot, \bar{w}_{i}\right)\right)^{*}+\operatorname{epi} \delta_{C}^{*}
$$

By Proposition 2.2, we obtain

$$
\begin{aligned}
(0,0) \in & \bigcup_{\epsilon_{0}^{1} \geqq 0}\left\{\left(\xi_{0}^{1},\left\langle\xi_{0}^{1}, \bar{x}\right\rangle+\epsilon_{0}^{1}-f(\bar{x}, \bar{u})\right) \mid \xi_{0}^{1} \in \partial_{\epsilon_{0}^{1}}(f(\cdot, \bar{u}))(\bar{x})\right\} \\
& +\bigcup_{\epsilon_{0}^{2} \geqq 0}\left\{\left(\xi_{0}^{2},\left|\xi_{0}^{2}, \bar{x}\right\rangle+\epsilon_{0}^{2}+\bar{r} g(\bar{x}, \bar{v})\right) \mid \xi_{0}^{2} \in \partial_{\epsilon_{0}^{2}}(-\bar{r} g(\cdot, \bar{v}))(\bar{x})\right\}
\end{aligned}
$$




$$
\begin{aligned}
& +\bigcup_{\epsilon^{*} \geqq 0}\left\{\left(\xi^{*},\left\langle\xi^{*}, \bar{x}\right\rangle+\epsilon^{*}-\sum_{i=1}^{m} \bar{\lambda}_{i} h_{i}\left(\bar{x}, \bar{w}_{i}\right)\right) \mid \xi^{*} \in \partial_{\epsilon^{*}}\left(\sum_{i=1}^{m} \bar{\lambda}_{i} h_{i}\left(\cdot, \bar{w}_{i}\right)\right)(\bar{x})\right\} \\
& +\bigcup_{\epsilon_{m+1} \geqq 0}\left\{\left(\xi_{m+1},\left\langle\xi_{m+1}, \bar{x}\right\rangle+\epsilon_{m+1}-\delta_{C}(\bar{x})\right) \mid \xi_{m+1} \in \partial_{\epsilon_{m+1}} \delta_{C}(\bar{x})\right\} .
\end{aligned}
$$

So, there exist $\bar{\xi}_{0}^{1} \in \partial_{\epsilon_{0}^{1}}(f(\cdot, \bar{u}))(\bar{x}), \bar{\xi}_{0}^{2} \in \partial_{\epsilon_{0}^{2}}(-\bar{r} g(\cdot, \bar{v}))(\bar{x}), \bar{\xi}^{*} \in \partial_{\epsilon^{*}}\left(\sum_{i=1}^{m} \bar{\lambda}_{i} h_{i}\left(\cdot, \bar{w}_{i}\right)\right)(\bar{x}), \bar{\xi}_{m+1} \in$ $\partial_{\epsilon_{m+1}} \delta_{C}(\bar{x}), \epsilon_{0}^{1} \geqq 0, \epsilon_{0}^{2} \geqq 0, \epsilon^{*} \geqq 0$, and $\epsilon_{m+1} \geqq 0$ such that

$$
0=\bar{\xi}_{0}^{1}+\bar{\xi}_{0}^{2}+\bar{\xi}^{*}+\bar{\xi}_{m+1} \quad \text { and } \quad \epsilon_{0}^{1}+\epsilon_{0}^{2}+\epsilon^{*}+\epsilon_{m+1}=f(\bar{x}, \bar{u})-\bar{r} g(\bar{x}, \bar{v})+\sum_{i=1}^{m} \bar{\lambda}_{i} h_{i}\left(\bar{x}, \bar{w}_{i}\right)
$$

By Proposition 2.5, there exist $\bar{\xi}_{0}^{1} \in \partial_{\epsilon_{0}^{1}}(f(\cdot, \bar{u}))(\bar{x}), \bar{\xi}_{0}^{2} \in \partial_{\epsilon_{0}^{2}}(-\bar{r} g(\cdot, \bar{v}))(\bar{x}), \bar{\xi}_{i} \in \partial_{\epsilon_{i}}\left(\bar{\lambda}_{i} h_{i}(\cdot\right.$, $\left.\left.\bar{w}_{i}\right)\right)(\bar{x}), \bar{\xi}_{m+1} \in \partial_{\epsilon_{m+1}} \delta_{C}(\bar{x}), \epsilon_{0}^{1} \geqq 0, \epsilon_{0}^{2} \geqq 0, \epsilon_{i} \geqq 0, i=1, \ldots, m$, and $\epsilon_{m+1} \geqq 0$ such that

$$
\begin{aligned}
& 0 \in \partial_{\epsilon_{0}^{1}}(f(\cdot, \bar{u}))(\bar{x})+\partial_{\epsilon_{0}^{2}}(-\bar{r} g(\cdot, \bar{v}))(\bar{x}) \\
& +\sum_{i=1}^{m} \partial_{\epsilon_{i}}\left(\bar{\lambda}_{i} h_{i}\left(\cdot, \bar{w}_{i}\right)\right)(\bar{x})+N_{C}^{\epsilon_{m+1}}(\bar{x}) \quad \text { and } \\
& \epsilon_{0}^{1}+\epsilon_{0}^{2}+\sum_{i=1}^{m+1} \epsilon_{i}=f(\bar{x}, \bar{u})-\bar{r} g(\bar{x}, \bar{v})+\sum_{i=1}^{m} \bar{\lambda}_{i} h_{i}\left(\bar{x}, \bar{w}_{i}\right) .
\end{aligned}
$$

Since $\bar{r}=\max _{(u, v) \in \mathcal{U} \times \mathcal{V}} \frac{f(\bar{x}, u)}{g(\bar{x}, v)}-\epsilon$, we have $\max _{u \in \mathcal{U}} f(\bar{x}, u)-\bar{r} \min _{v \in \mathcal{V}} g(\bar{x}, v)=\epsilon \min _{v \in \mathcal{V}} g(\bar{x}, v)$. So, we have

$$
f(\bar{x}, \bar{u})-\bar{r} g(\bar{x}, \bar{v}) \leqq \max _{u \in \mathcal{U}} f(\bar{x}, u)-\bar{r} \min _{v \in \mathcal{V}} g(\bar{x}, v)=\epsilon \min _{v \in \mathcal{V}} g(\bar{x}, v)
$$

So, the condition (13) holds. Also, from (15) and (16), we have

$$
\begin{gathered}
0 \in \partial_{\epsilon_{0}^{1}}\left(\max _{u \in \mathcal{U}} f(\cdot, u)\right)(\bar{x})+\partial_{\epsilon_{0}^{2}}\left(-\bar{r} \min _{v \in \mathcal{V}} g(\cdot, v)\right)(\bar{x}) \\
+\sum_{i=1}^{m} \partial_{\epsilon_{i}}\left(\bar{\lambda}_{i} h_{i}\left(\cdot, \bar{w}_{i}\right)\right)(\bar{x})+N_{C}^{\epsilon_{m+1}}(\bar{x}) \text { and } \\
\epsilon_{0}^{1}+\epsilon_{0}^{2}+\sum_{i=1}^{m+1} \epsilon_{i}-\epsilon \min _{v \in \mathcal{V}} g(\bar{x}, v) \leqq \sum_{i=1}^{m} \bar{\lambda}_{i} h_{i}\left(\bar{x}, \bar{w}_{i}\right) .
\end{gathered}
$$

Consequently, the conditions (12) and (14) hold.

$[(i i) \Rightarrow($ i) $]$ Taking account of the converse of the process for proving (i) $\Rightarrow$ (ii), we can easily check that the statement (ii) $\Rightarrow$ (i) holds.

Remark 3.3 Assume that $f: \mathbb{R}^{n} \times \mathbb{R}^{p} \rightarrow \mathbb{R}$ and $g: \mathbb{R}^{n} \times \mathbb{R}^{p} \rightarrow \mathbb{R}$ are functions such that, for all $x \in \mathbb{R}^{n}, f(x, \cdot)$, and $g(x, \cdot)$ are concave and convex, respectively. Then we know that Theorem 3.2 is equivalent to Theorem 3.3 from Lemma 3.1, immediately. 


\section{$4 \epsilon$-Duality theorems}

Following the approach in [13], we formulate a dual problem (RFD) for (RFP) as follows:

(RFD) $\max r$

$$
\begin{array}{ll}
\text { s.t. } 0 \in \partial_{\epsilon_{0}^{1}}\left(\max _{u \in \mathcal{U}} f(\cdot, u)\right)(x)+\partial_{\epsilon_{0}^{2}}\left(-r \min _{v \in \mathcal{V}} g(\cdot, v)\right)(x) \\
\\
+\sum_{i=1}^{m} \partial_{\epsilon_{i}}\left(\lambda_{i} h_{i}\left(\cdot, w_{i}\right)\right)(x)+N_{C}^{\epsilon_{m+1}}(x), \\
\max _{u \in \mathcal{U}} f(x, u)-r \min _{v \in \mathcal{V}} g(x, v) \geqq \epsilon \min _{v \in \mathcal{V}} g(x, v), \\
\epsilon_{0}^{1}+\epsilon_{0}^{2}+\sum_{i=1}^{m+1} \epsilon_{i}-\epsilon \min _{v \in \mathcal{V}} g(x, v) \leqq \sum_{i=1}^{m} \lambda_{i} h_{i}\left(x, w_{i}\right), \\
r \geqq 0, w_{i} \in \mathcal{W}_{i}, \lambda_{i} \geqq 0, i=1, \ldots, m, \\
\epsilon_{0}^{1} \geqq 0, \epsilon_{0}^{2} \geqq 0, \epsilon_{i} \geqq 0, i=1, \ldots, m+1 .
\end{array}
$$

Clearly,

$$
\begin{aligned}
F:= & \left\{(x, w, \lambda, r) \mid 0 \in \partial_{\epsilon_{0}^{1}}\left(\max _{u \in \mathcal{U}} f(\cdot, u)\right)(x)+\partial_{\epsilon_{0}^{2}}\left(-r \min _{v \in \mathcal{V}} g(\cdot, v)\right)(x)\right. \\
& +\sum_{i=1}^{m} \partial_{\epsilon_{i}}\left(\lambda_{i} h_{i}\left(\cdot, w_{i}\right)\right)(x)+N_{\mathbb{R}_{+}}^{\epsilon_{2}}(x), \max _{u \in \mathcal{U}} f(x, u)-r \min _{v \in \mathcal{V}} g(x, v) \geqq \epsilon g(x, v), \\
& \epsilon_{0}^{1}+\epsilon_{0}^{2}+\sum_{i=1}^{m+1} \epsilon_{i}-\epsilon \min _{v \in \mathcal{V}} g(x, v) \leqq \sum_{i=1}^{m} \lambda_{i} h_{i}\left(x, w_{i}\right), \\
& \left.r \geqq 0, w_{i} \in \mathcal{W}_{i}, \lambda_{i} \geqq 0, \epsilon_{0}^{1} \geqq 0, \epsilon_{0}^{2} \geqq 0, \epsilon_{i} \geqq 0, i=1, \ldots, m, \epsilon_{m+1} \geqq 0\right\}
\end{aligned}
$$

is the feasible set of (RFD).

Let $\epsilon \geqq 0$. Then $(\bar{x}, \bar{w}, \bar{\lambda}, \bar{r})$ is called an $\epsilon$-solution of (RFD) if, for any $(y, w, \lambda, r) \in F, \bar{r} \geqq$ $r-\epsilon$.

When $\epsilon=0, \max _{u \in \mathcal{U}} f(x, u)=f(x), \min _{v \in \mathcal{V}} g(x, v)=g(x)$, and $h_{i}\left(x, w_{i}\right)=h_{i}(x), i=1, \ldots, m$, (RFP) becomes (FP), and (RFD) collapses to the Mond-Weir type dual problem (FD) for (FP) as follows [28]:

(FD)

$$
\begin{array}{ll}
\max & \\
\text { s.t. } & 0 \in \partial f(x)+\partial(-r g)(x)+\sum_{i=1}^{m} \partial \lambda_{i} h_{i}(x)+N_{C}(x), \\
& f(x)-r g(x) \geqq 0, \lambda_{i} h_{i}(x) \geqq 0, \\
& r \geqq 0, \lambda_{i} \geqq 0, i=1, \ldots, m .
\end{array}
$$

Now, we prove $\epsilon$-weak and $\epsilon$-strong duality theorems which hold between (RFP) and (RFD). 
Theorem 4.1 ( $\epsilon$-Weak duality theorem) For any feasible $x$ of (RFP) and any feasible $(y, w, \lambda, r)$ of (RFD),

$$
\max _{(u, v) \in \mathcal{U} \times \mathcal{V}} \frac{f(x, u)}{g(x, v)} \geqq r-\epsilon .
$$

Proof Let $x$ and $(y, w, \lambda, r)$ be feasible solutions of (RFP) and (RFD), respectively. Then there exist $\bar{\xi}_{0}^{1} \in \partial_{\epsilon_{0}^{1}}\left(\max _{u \in \mathcal{U}} f(\cdot, u)\right)(y), \bar{\xi}_{0}^{2} \in \partial_{\epsilon_{0}^{2}}\left(-r \min _{v \in \mathcal{V}} g(\cdot, v)\right)(y), \bar{\xi}_{i} \in \partial_{\epsilon_{i}}\left(\lambda_{i} h_{i}\left(\cdot, w_{i}\right)\right)(y)$, $\bar{\xi}_{m+1} \in N_{C}^{\epsilon_{m+1}}(y), \epsilon_{0}^{1} \geqq 0, \epsilon_{0}^{2} \geqq 0, \epsilon_{i} \geqq 0, i=1, \ldots, m$, and $\epsilon_{m+1} \geqq 0$ such that

$$
\begin{aligned}
& \bar{\xi}_{0}^{1}+\bar{\xi}_{0}^{2}+\sum_{i=1}^{m+1} \bar{\xi}_{i}=0, \quad \max _{u \in \mathcal{U}} f(y, u)-r \min _{v \in \mathcal{V}} g(y, v) \geqq \epsilon \min _{v \in \mathcal{V}} g(y, v) \quad \text { and } \\
& \epsilon_{0}^{1}+\epsilon_{0}^{2}+\sum_{i=1}^{m+1} \epsilon_{i}-\epsilon \min _{v \in \mathcal{V}} g(y, v) \leqq \sum_{i=1}^{m} \lambda_{i} h_{i}\left(y, w_{i}\right) .
\end{aligned}
$$

Thus, we have

$$
\begin{aligned}
\max _{u \in \mathcal{U}} f(x, u)-r \min _{v \in \mathcal{V}} g(x, v)+\epsilon \min _{v \in \mathcal{V}} g(x, v) \\
\geqq \max _{u \in \mathcal{U}} f(y, u)-r \min _{v \in \mathcal{V}} g(y, v)+\left\langle\bar{\xi}_{0}^{1}+\bar{\xi}_{0}^{2}, x-y\right\rangle-\epsilon_{0}^{1}-\epsilon_{0}^{2}+\epsilon \min _{v \in \mathcal{V}} g(x, v) \\
=\max _{u \in \mathcal{U}} f(y, u)-r \min _{v \in \mathcal{V}} g(y, v)-\left\langle\sum_{i=1}^{m+1} \bar{\xi}_{i}, x-y\right\rangle-\epsilon_{0}^{1}-\epsilon_{0}^{2}+\epsilon \min _{v \in \mathcal{V}} g(x, v) \\
\geqq \max _{u \in \mathcal{U}} f(y, u)-r \min _{v \in \mathcal{V}} g(y, v)+\sum_{i=1}^{m} \lambda_{i} h_{i}\left(y, w_{i}\right)-\sum_{i=1}^{m} \lambda_{i} h_{i}\left(x, w_{i}\right)-\epsilon_{0}^{1}-\epsilon_{0}^{2}-\sum_{i=1}^{m+1} \epsilon_{i} \\
\quad+\epsilon \min _{v \in \mathcal{V}} g(x, v) \\
\geqq \max _{u \in \mathcal{U}} f(y, u)-r \min _{v \in \mathcal{V}} g(y, v)+\sum_{i=1}^{m} \lambda_{i} h_{i}\left(y, w_{i}\right)-\epsilon_{0}^{1}-\epsilon_{0}^{2}-\sum_{i=1}^{m+1} \epsilon_{i} \\
\geqq \max _{u \in \mathcal{U}} f(y, u)-r \min _{v \in \mathcal{V}} g(y, v)-\epsilon \min _{v \in \mathcal{V}} g(y, v) \\
\geqq 0 .
\end{aligned}
$$

Hence, we have $\max _{(u, v) \in \mathcal{U} \times \mathcal{V}} \frac{f(x, u)}{g(x, v)} \geqq r-\epsilon$.

Theorem 4.2 ( $\epsilon$-Strong duality theorem) Suppose that

$$
\bigcup_{w_{i} \in \mathcal{W}_{i}, \lambda_{i} \geq 0} \operatorname{epi}\left(\sum_{i=1}^{m} \lambda_{i} g_{i}\left(\cdot, w_{i}\right)\right)^{*}+C^{*} \times \mathbb{R}_{+}
$$

is closed. If $\bar{x}$ is an $\epsilon$-solution of (RFP) and $\max _{(u, v) \in \mathcal{U} \times \mathcal{V}} \frac{f(\bar{x}, u)}{g(\bar{x}, v)}-\epsilon \geqq 0$, then there exist $\bar{w} \in \mathbb{R}^{q}$, $\bar{\lambda} \in \mathbb{R}_{+}^{m}$, and $\bar{r} \in \mathbb{R}_{+}$such that $(\bar{x}, \bar{w}, \bar{\lambda}, \bar{r})$ is a $2 \epsilon$-solution of (RFD).

Proof Let $\bar{x} \in A$ be an $\epsilon$-solution of (RFP). Let $\bar{r}=\max _{(u, v) \in \mathcal{U} \times \mathcal{V}} \frac{f(\bar{x}, u)}{g(\bar{x}, v)}$. Then, by Theorem 3.2, there exist $\bar{w}_{i} \in \mathcal{W}_{i}, \bar{\lambda}_{i} \geqq 0, \epsilon_{0}^{1} \geqq 0, \epsilon_{0}^{2} \geqq 0, \epsilon_{i} \geqq 0, i=1, \ldots, m$, and $\epsilon_{m+1}$ such 
that

$$
\begin{aligned}
& 0 \in \partial_{\epsilon_{0}^{1}}\left(\max _{u \in \mathcal{U}} f(\cdot, u)\right)(\bar{x})+\partial_{\epsilon_{0}^{2}}\left(-\bar{r} \min _{v \in \mathcal{V}} g(\cdot, v)\right)(\bar{x})+\sum_{i=1}^{m} \partial_{\epsilon_{i}}\left(\bar{\lambda}_{i} h_{i}\left(\cdot, \bar{w}_{i}\right)\right)(\bar{x})+N_{C}^{\epsilon_{m}+1}(\bar{x}), \\
& \max _{u \in \mathcal{U}} f(\bar{x}, u)-\bar{r} \min _{v \in \mathcal{V}} g(\bar{x}, v)=\epsilon \min _{v \in \mathcal{V}} g(\bar{x}, v) \text { and } \\
& \epsilon_{0}^{1}+\epsilon_{0}^{2}+\sum_{i=1}^{m+1} \epsilon_{i}-\epsilon \min _{v \in \mathcal{V}} g(\bar{x}, v)=\sum_{i=1}^{m} \bar{\lambda}_{i} h_{i}\left(\bar{x}, \bar{w}_{i}\right) .
\end{aligned}
$$

So, $(\bar{x}, \bar{w}, \bar{\lambda}, \bar{r})$ is a feasible solution of (RFD). For any feasible $(y, u, v, w, \lambda, v)$ of (RFD), it follows from Theorem 4.1 ( $\epsilon$-weak duality theorem) that

$$
\bar{r}=\max _{(u, v) \in \mathcal{U} \times \mathcal{V}} \frac{f(\bar{x}, u)}{g(\bar{x}, v)}-\epsilon \geqq r-\epsilon-\epsilon=r-2 \epsilon .
$$

Thus $(\bar{x}, \bar{w}, \bar{\lambda}, \bar{r})$ is a $2 \epsilon$-solution of (RFD).

Remark 4.1 Using the optimality conditions of Theorem 3.2, robust fractional dual problem (RFD) for a robust fractional problem (RFP) in the convex constraint functions with uncertainty is formulated. However, when we formulated the dual problem using optimality condition in Theorem 3.3, we could not know whether $\epsilon$-weak duality theorem is established, or not. It is an open question.

Now we give an example illustrating our duality theorems.

Example 4.1 Consider the following fractional programming problem with uncertainty:

$$
\begin{aligned}
& \text { (RFP) } \min \max _{(u, v) \in \mathcal{U} \times \mathcal{V}} \frac{u x+1}{v x+2} \\
& \text { s.t. } 2 w_{1} x-3 \leqq 0, w_{1} \in[1,2], \\
& x \in \mathbb{R}_{+},
\end{aligned}
$$

where $\mathcal{U}=[1,2]$ and $\mathcal{V}=[1,2]$

Now we transform the problem (RFP) into the robust non-fractional convex optimization problem $(\mathrm{RNCP})_{r}$ with a parametric $r \in \mathbb{R}_{+}$:

$$
\begin{gathered}
(\mathrm{RNCP})_{r} \quad \min \max _{u \in[1,2]}(u x+1)-r \min _{v \in[1,2]}(v x+2) \\
\text { s.t. } 2 w_{1} x-3 \leqq 0, w_{1} \in[1,2], \\
x \in \mathbb{R}_{+} .
\end{gathered}
$$

Let $f(x, u)=u x+1, g(x, v)=v x+2, h_{1}\left(x, w_{1}\right)=-2 w_{1} x-3$, and $\epsilon \in\left[0, \frac{9}{22}\right]$. Then $A:=\{x \in$ $\left.\mathbb{R} \mid 0 \leqq x \leqq \frac{3}{4}\right\}$ is the set of all robust feasible solutions of (RFP) and $\bar{A}:=\{x \in \mathbb{R} \mid 0 \leqq x \leqq$ $\left.\frac{4 \epsilon}{3-2 \epsilon}\right\}$ is the set of all $\epsilon$-solutions of (RFP). Let $F:=\left\{\left(y, w_{1}, \lambda_{1}, r\right) \mid 0 \in \partial_{\epsilon_{0}^{1}}\left(\max _{u \in \mathcal{U}} f(\cdot, u)\right)(y)+\right.$ $\partial_{\epsilon_{0}^{2}}\left(-r \min _{v \in \mathcal{V}} g(\cdot, v)\right)(y)+\partial_{\epsilon_{1}}\left(\lambda_{1} h_{1}\left(\cdot, w_{1}\right)\right)(y)+N_{\mathbb{R}_{+}}^{\epsilon_{2}}(x), \max _{u \in \mathcal{U}} f(y, u)-r \min _{v \in \mathcal{V}} g(y, v) \geqq$ 
$\epsilon \min _{v \in \mathcal{V}} g(y, v), \epsilon_{0}^{1}+\epsilon_{0}^{2}+\epsilon_{1}+\epsilon_{2}-\epsilon \min _{v \in \mathcal{V}} g(y, v) \leqq \lambda_{1} h_{1}\left(y, w_{1}\right), r \geqq 0, w_{1} \in[1,2], \lambda_{1} \geqq 0, \epsilon_{0}^{1} \geqq$ $\left.0, \epsilon_{0}^{2} \geqq 0, \epsilon_{1} \geqq 0, \epsilon_{2} \geqq 0\right\}$. Then we formulate a dual problem (RFD) for (RFP) as follows:

(RFD) $\max r$

s.t. $\left(y, w_{1}, \lambda_{1}, r\right) \in F$.

Then $F$ is the set of all robust feasible solutions of (RFD).

Now we calculate the set $F=\widetilde{A} \cup \widetilde{B}$.

$$
\begin{aligned}
& \widetilde{A}:=\left\{\left(0, w_{1}, \lambda_{1}, r\right) \mid 0 \in \partial_{\epsilon_{0}^{1}}\left(\max _{u \in \mathcal{U}} f(\cdot, u)\right)(0)\right. \\
& +\partial_{\epsilon_{0}^{2}}\left(-r \min _{v \in \mathcal{V}} g(\cdot, v)\right)(0)+\partial_{\epsilon_{1}}\left(\lambda_{1} h_{1}\left(\cdot, w_{1}\right)\right)(0) \\
& +N_{\mathbb{R}_{+}}^{\epsilon_{2}}(0), \max _{u \in \mathcal{U}} f(0, u)-r \min _{v \in \mathcal{V}} g(0, v) \geqq \epsilon \min _{v \in \mathcal{V}} g(0, v), \epsilon_{0}^{1}+\epsilon_{0}^{2}+\epsilon_{1}+\epsilon_{2}-\epsilon \min _{v \in \mathcal{V}} g(0, v) \\
& \left.\leqq \lambda_{1} h_{1}\left(0, w_{1}\right), r \geqq 0, u \in[1,2], \lambda_{1} \geqq 0, \epsilon_{0}^{1} \geqq 0, \epsilon_{0}^{2} \geqq 0, \epsilon_{1} \geqq 0, \epsilon_{2} \geqq 0\right\} \\
& =\left\{\left(0, w_{1}, \lambda_{1}, r\right) \mid 0 \in\{2\}+\{-r\}+\left\{2 \lambda_{1} w_{1}\right\}+(-\infty, 0], 1-2 r \geqq 2 \epsilon, \epsilon_{0}^{1}+\epsilon_{0}^{2}+\epsilon_{1}+\epsilon_{2}\right. \\
& \left.-2 \epsilon \leqq-3 \lambda_{1}, r \geqq 0, w_{1} \in[1,2], \lambda_{1} \geqq 0, \epsilon_{0}^{1} \geqq 0, \epsilon_{0}^{2} \geqq 0, \epsilon_{1} \geqq 0, \epsilon_{2} \geqq 0\right\} \\
& =\left\{\left(0, w_{1}, \lambda_{1}, r\right) \mid r \leqq 2+2 \lambda_{1} w_{1}, r \leqq \frac{1-2 \epsilon}{2}, \epsilon_{0}^{1}+\epsilon_{0}^{2}+\epsilon_{1}+\epsilon_{2}-2 \epsilon \leqq-3 \lambda_{1}, r \geqq 0\right. \text {, } \\
& \left.w_{1} \in[1,2], \lambda_{1} \geqq 0, \epsilon_{0}^{1} \geqq 0, \epsilon_{0}^{2} \geqq 0, \epsilon_{1} \geqq 0, \epsilon_{2} \geqq 0\right\}, \\
& \widetilde{B}:=\left\{\left(y, w_{1}, \lambda_{1}, r\right) \mid 0 \in \partial_{\epsilon_{0}^{1}}\left(\max _{u \in \mathcal{U}} f(\cdot, u)\right)(y)+\partial_{\epsilon_{0}^{2}}\left(-r \min _{v \in \mathcal{V}} g(\cdot, v)\right)(y)+\partial_{\epsilon_{1}}\left(\lambda_{1} h_{1}\left(\cdot, w_{1}\right)\right)(y)\right. \\
& +N_{\mathbb{R}_{+}}^{\epsilon_{2}}(y), \max _{u \in \mathcal{U}} f(y, u)-r \min _{v \in \mathcal{V}} g(y, v) \geqq \epsilon \min _{v \in \mathcal{V}} g(y, v), \epsilon_{0}^{1}+\epsilon_{0}^{2}+\epsilon_{1}+\epsilon_{2}-\epsilon \min _{v \in \mathcal{V}} g(y, v) \\
& \left.\leqq \lambda_{1} h_{1}\left(y, w_{1}\right), y>0, r \geqq 0, w_{1} \in[1,2], \lambda_{1} \geqq 0, \epsilon_{0}^{1} \geqq 0, \epsilon_{0}^{2} \geqq 0, \epsilon_{1} \geqq 0, \epsilon_{2} \geqq 0\right\} \\
& =\left\{\left(y, w_{1}, \lambda_{1}, r\right) \mid 0 \in\{2\}+\{-r\}+\left\{2 \lambda_{1} w_{1}\right\}+\left[-\frac{\epsilon_{2}}{y}, 0\right], 2 y+1-r(y+2) \geqq \epsilon(y+2)\right. \text {, } \\
& y>0, \epsilon_{0}^{1}+\epsilon_{0}^{2}+\epsilon_{1}+\epsilon_{2}-\epsilon(y+2) \leqq \lambda_{1}\left(2 w_{1} y-3\right), r \geqq 0, w_{1} \in[1,2], \lambda_{1} \geqq 0, \\
& \left.\epsilon_{0}^{1} \geqq 0, \epsilon_{0}^{2} \geqq 0, \epsilon_{1} \geqq 0, \epsilon_{2} \geqq 0\right\} \\
& =\left\{\left(y, w_{1}, \lambda_{1}, r\right) \mid 0 \in\left[2-r+2 \lambda_{1} w_{1}-\frac{\epsilon_{2}}{y}, 2-r+2 \lambda_{1} w_{1}\right], 2 y+1-r(y+2) \geqq\right. \\
& \epsilon(y+2), \epsilon_{0}^{1}+\epsilon_{0}^{2}+\epsilon_{1}+\epsilon_{2}-\epsilon(y+2) \leqq \lambda_{1}\left(2 w_{1} y-3\right), y>0, r \geqq 0, w_{1} \in[1,2], \\
& \left.\lambda_{1} \geqq 0, \epsilon_{0}^{1} \geqq 0, \epsilon_{0}^{2} \geqq 0, \epsilon_{1} \geqq 0, \epsilon_{2} \geqq 0\right\} \text {. }
\end{aligned}
$$

We can check for any $x \in A$ and any $\left(y, w_{1}, \lambda_{1}, r\right) \in F$,

$$
\max _{(u, v) \in \mathcal{U} \times \mathcal{V}} \frac{f(x, u)}{g(x, v)} \geqq r-\epsilon,
$$


that is, $\epsilon$-weak duality holds. Indeed, let $x \in A$ and $\left(y, w_{1}, \lambda_{1}, r\right) \in \widetilde{A}$ be fixed. Then

$$
\begin{aligned}
& \max _{u \in[1,2]} f(x, u)-r \min _{v \in[1,2]} g(x, v)+\epsilon \min _{v \in[1,2]} g(x, v) \\
& \quad=2 x+1-r(x+2)+\epsilon(x+2) \\
& \quad=(2-r) x+1-2 r+\epsilon(x+2) \\
& \quad \geqq-2 \lambda_{1} w_{1} x+2 \epsilon+\epsilon(x+2) \\
& \quad \geqq-3 \lambda_{1}+2 \epsilon+\epsilon(x+2) \\
& \quad \geqq-3 \lambda_{1}+\epsilon_{0}^{1}+\epsilon_{0}^{2}+\epsilon_{1}+\epsilon_{2}+3 \lambda_{1}+\epsilon(x+2) \\
& \quad \geqq 0 .
\end{aligned}
$$

Moreover, let $x \in A$ and $\left(y, u, v, w_{1}, \lambda_{1}, r\right) \in \widetilde{B}$ be fixed.

$$
\begin{aligned}
& \max _{u \in[1,2]} f(x, u)-r \min _{v \in[1,2]} g(x, v)+\epsilon \min _{v \in[1,2]} g(x, v) \\
& \quad=2 x+1-r(x+2)+\epsilon(x+2) \\
& \quad=2 y+1-r(y+2)+(2-r)(x-y)+\epsilon(x+2) .
\end{aligned}
$$

If $x-y \geqq 0$, then

$$
\begin{aligned}
& \max _{u \in[1,2]} f(x, u)-r \min _{v \in[1,2]} g(x, v)+\epsilon \min _{v \in[1,2]} g(x, v) \\
& \quad=2 y+1-r(y+2)+(2-r)(x-y)+\epsilon(x+2) \\
& \quad \geqq 2 y+1-r(y+2)-2 \lambda_{1} w_{1}(x-y)+\epsilon(x+2) \\
& \quad \geqq 2 y+1-r(y+2)+2 \lambda_{1} w_{1} y-2 \lambda_{1} w_{1} x+\epsilon(x+2) \\
& \geqq \epsilon(y+2)+2 \lambda_{1} w_{1} y-3 \lambda_{1}+\epsilon(x+2) \\
& \geqq \epsilon_{0}^{1}+\epsilon_{0}^{2}+\epsilon_{1}+\epsilon_{2}+3 \lambda_{1}-3 \lambda_{1}+\epsilon(x+2) \\
& \geqq 0 .
\end{aligned}
$$

If $x-y<0$, then

$$
\begin{aligned}
& \max _{u \in[1,2]} f(x, u)-r \min _{v \in[1,2]} g(x, v)+\epsilon \min _{v \in[1,2]} g(x, v) \\
& \quad=2 y+1-r(y+2)+(2-r)(x-y)+\epsilon(x+2) \\
& \geqq 2 y+1-r(y+2)+\left(-2 \lambda_{1} w_{1}+\frac{\epsilon_{2}}{y}\right)(x-y)+\epsilon(x+2) \\
& \geqq 2 y+1-r(y+2)+2 \lambda_{1} w_{1} y-\epsilon_{2}-2 \lambda_{1} w_{1} x+\frac{\epsilon_{2}}{y} x+\epsilon(x+2) \\
& \geqq \epsilon(y+2)+2 \lambda_{1} w_{1} y-\epsilon_{2}-3 \lambda_{1}+\frac{\epsilon_{2}}{y} x+\epsilon(x+2) \\
& \geq \epsilon_{0}^{1}+\epsilon_{0}^{2}+\epsilon_{1}+\epsilon_{2}+3 \lambda_{1}-\epsilon_{2}-3 \lambda_{1}+\frac{\epsilon_{2}}{y} x+\epsilon(x+2) \\
& \geqq 0 .
\end{aligned}
$$


Let $\epsilon=\frac{1}{3}$. Then $\bar{x} \in \bar{A}:=\left\{x \in \mathbb{R} \mid 0 \leqq x \leqq \frac{4}{7}\right\}$ is the set of all $\epsilon$-solutions of (RFP) and $\frac{1}{6} \leqq \bar{r} \leqq \frac{1}{2}$.

If $\bar{x}=0$, then $\bar{r}=\frac{1}{6}$. When $\epsilon=\frac{1}{3}$, we can calculate the set $\tilde{A}$ as follows:

$$
\tilde{A}:=\left\{\left(0, w_{1}, \lambda_{1}, r\right) \mid 0 \leqq r \leqq \frac{1}{6}, 0 \leqq \lambda_{1} \leqq \frac{2}{9}, w_{1} \in[1,2]\right\} .
$$

Let $\bar{w}_{1}=2, \bar{\lambda}_{1}=\frac{1}{9}$. Then $\left(0,2, \frac{5}{8}, \frac{1}{9}\right) \in \tilde{A}$. So, we have

$$
\bar{r}=\max _{(u, v) \in \mathcal{U} \times \mathcal{V}} \frac{f(\bar{x}, u)}{g(\bar{x}, v)}-\epsilon=\frac{1}{6}=\frac{5}{6}-2 \epsilon \geqq r-2 \epsilon .
$$

Hence, $\left(0,2, \frac{1}{9}, \frac{1}{6}\right)$ is a $2 \epsilon$-solution of (RFD). So, $\epsilon$-strong duality holds. If $0<\bar{x} \leqq \frac{4}{7}$, then $\frac{1}{6}<\bar{r} \leqq \frac{1}{2}$. When $\epsilon=\frac{1}{3}$, we can calculate the set $\tilde{B}$ as follows:

$$
\begin{aligned}
\widetilde{B}:= & \left\{\left(y, w_{1}, \lambda_{1}, r\right) \mid y>0,2+2 \lambda_{1} w_{1}-\frac{\epsilon_{2}}{y} \leqq r \leqq \frac{5 y+1}{3(y+2)}, \epsilon_{2}-\frac{1}{3}(y+2) \leqq\right. \\
& \left.\lambda_{1}\left(2 w_{1} y-3\right), r \geqq 0, u \in[1,2], v \in[1,2], w_{1} \in[1,2], \epsilon_{2} \geqq 0\right\} .
\end{aligned}
$$

Let $\bar{w}_{1}=2, \bar{\lambda}_{1}=0$, and $\epsilon_{2}=\frac{\bar{x}+2}{3}$. Then $\left(\bar{x}, 2,0, \frac{5 \bar{x}+1}{3(\bar{x}+2)}\right) \in \tilde{B}$. So, we have

$$
\bar{r}=\max _{(u, v) \in \mathcal{U} \times \mathcal{V}} \frac{f(\bar{x}, u)}{g(\bar{x}, v)}-\epsilon=\frac{5 \bar{x}+1}{3(\bar{x}+2)} \geqq r \geqq r-2 \epsilon .
$$

Hence, $\left(\bar{x}, 2,0, \frac{5 \bar{x}+1}{3(\bar{x}+2)}\right)$ is a $2 \epsilon$-solution of (RFD). So, $\epsilon$-strong duality holds.

\section{Competing interests}

The authors declare that they have no competing interests.

\section{Authors' contributions}

All authors contributed equally in this paper and they read and approved the final manuscript.

\section{Acknowledgements}

The authors are grateful to the referees and the editor for their valuable comments and constructive suggestions, which have contributed to the final version of the paper. This research was supported by Basic Science Research Program through the National Research Foundation of Korea (NRF) funded by the Ministry of Education (NRF-2013R1A1A2005378).

Received: 29 April 2014 Accepted: 28 November 2014 Published: 16 Dec 2014

\section{References}

1. Govil, MG, Mehra, A: $\epsilon$-Optimality for multiobjective programming on a Banach space. Eur. J. Oper. Res. 157(1), 106-112 (2004)

2. Gutiérrez, C, Jiménez, B, Novo, V: Multiplier rules and saddle-point theorems for Helbig's approximate solutions in convex Pareto problems. J. Glob. Optim. 32(3), 367-383 (2005)

3. Hamel, A: An $\epsilon$-Lagrange multiplier rule for a mathematical programming problem on Banach spaces. Optimization 49(1-2), 137-149 (2001)

4. Liu, JC: $\epsilon$-Duality theorem of nondifferentiable nonconvex multiobjective programming. J. Optim. Theory Appl. 69(1), 153-167 (1991)

5. Liu, JC: $\epsilon$-Pareto optimality for nondifferentiable multiobjective programming via penalty function. J. Math. Anal. Appl. 198(1), 248-261 (1996)

6. Strodiot, JJ, Nguyen, VH, Heukemes, N: $\epsilon$-Optimal solutions in nondifferentiable convex programming and some related question. Math. Program. 25(3), 307-328 (1983)

7. Yokoyama, K: Epsilon approximate solutions for multiobjective programming problems. J. Math. Anal. Appl. 203(1), 142-149 (1996)

8. Lee, GM, Lee, JH: $\epsilon$-Duality for convex semidefinite optimization problem with conic constraints. J. Inequal. Appl. 2010, Article ID 363012 (2010) 
9. Lee, JH, Lee, GM: On $\epsilon$-solutions for convex optimization problems with uncertainty data. Positivity 16(3), 509-526 (2012)

10. Barros, A, Frenk, JBG, Schaible, S, Zhang, S: Using duality to solve generalized fractional programming problems J. Glob. Optim. 8(2), 139-170 (1996)

11. Chinchuluun, A, Yuan, D, Pardalos, PM: Optimality conditions and duality for nondifferentiable multiobjective fractional programming with generalized convexity. Ann. Oper. Res. 154(1), 133-147 (2007)

12. Frenk, JBG, Schaible, S: Fractional programming. In: Handbook of Generalized Convexity and Monotonicity, pp. 333-384. Springer, Berlin (2004)

13. Gupta, P, Mehra, A, Shiraishi, S, Yokoyama, K: $\epsilon$-Optimality for minimax programming problems. J. Nonlinear Convex Anal. 7(2), 277-288 (2006)

14. Gupta, P, Shiraishi, S, Yokoyama, K: $\epsilon$-Optimality without constraint qualification for multibojective fractional program. J. Nonlinear Convex Anal. 6(2), 347-357 (2005)

15. Liang, ZA, Huang, HX, Pardalos, PM: Optimality conditions and duality for a class of nonlinear fractional programming problems. J. Optim. Theory Appl. 110(3), 611-619 (2001)

16. Beck, A, Ben-Tal, A: Duality in robust optimization: primal worst equals dual best. Oper. Res. Lett. 37(1), 1-6 (2009)

17. Ben-Tal, A, Ghaoui, LE, Nemirovski, A: Robust Optimization. Princeton Series in Applied Mathematics. Princeton University Press, Princeton (2009)

18. Ben-Tal, A, Nemirovski, A: A selected topics in robust convex optimization. Math. Program., Ser. B 112(1), 125-158 (2008)

19. Bersimas, D, Brown, D: Constructing uncertainty sets for robust linear optimization. Oper. Res. 57(6), 1483-1495 (2009)

20. Bersimas, D, Pachamanova, D, Sim, M: Robust linear optimization under general norms. Oper. Res. Lett. 32(6), 510-516 (2004)

21. Jeyakumar, V, Li, GY: Strong duality in robust convex programming: complete characterizations. SIAM J. Optim. 20(6), 3384-3407 (2010)

22. Jeyakumar, V, Li, GY: Robust duality for fractional programming problems with constraint-wise data uncertainty. J. Optim. Theory Appl. 151(2), 292-303 (2011)

23. Hiriart-Urruty, JB, Lemarechal, C: Convex Analysis and Minimization Algorithms, vols. I and II. Springer, Berlin (1993)

24. Jeyakumar, V: Asymptotic dual conditions characterizing optimality for convex programs. J. Optim. Theory Appl. 93(1), 153-165 (1997)

25. Jeyakumar, V, Lee, GM, Dinh, N: New sequential Lagrange multiplier conditions characterizing optimality without constraint qualification for convex programs. SIAM J. Optim. 14(2), 534-547 (2003)

26. Jeyakumar, V, Lee, GM, Dinh, N: Characterization of solution sets of convex vector minimization problems. Eur. J. Oper. Res. 174(3), 1380-1395 (2006)

27. Rockafellar, RT: Convex Analysis. Princeton University Press, Princeton (1970)

28. Liu, JC, Kimura, Y, Tanaka, K: Three types dual model for minimax fractional programming. Comput. Math. Appl. 38(7-8), 143-155 (1999)

\section{Submit your manuscript to a SpringerOpen ${ }^{\ominus}$ journal and benefit from:}

- Convenient online submission

Rigorous peer review

- Immediate publication on acceptance

- Open access: articles freely available online

- High visibility within the field

- Retaining the copyright to your article 